\title{
The Structure of Connectivity Functions
}

\author{
Songbao Mo
}

\section{VICTORIA UNIVERSITY OF WELLINGTON}

Te Whare Wānanga o te Ūpoko o te Ika a Māui

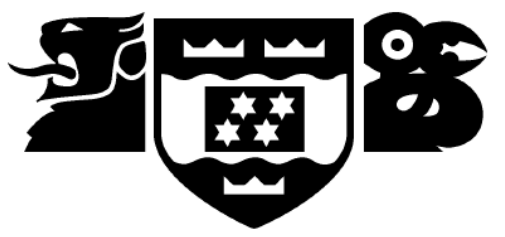

School of Mathematics, Statistics

and Operations Research

Te Kura Mātai Tatauranga, Rangahau Pūnaha

A thesis

submitted to the Victoria University of Wellington in fulfilment of the requirements for the degree of

Master of Science

in Mathematics.

Victoria University of Wellington

2014 


\begin{abstract}
Graphs, matroids and polymatroids all have associated connectivity functions, and many properties of these structures follow from properties of their connectivity functions. This motivates the study of connectivity functions in general. It turns out that connectivity functions are surprisingly highly structured. We prove some interesting results about connectivity functions. In particular we show that every connectivity function is a connectivity function of a half-integral polymatroid.
\end{abstract}




\section{Acknowledgements}

I would like to thank Geoff Whittle for his advise and supervision. I would also like to thank those who have helped and supported me during the study. 


\section{Contents}

$\begin{array}{lll}1 & \text { Introduction } & 1\end{array}$

2 Connectivity Functions 4

2.1 Connectivity functions . . . . . . . . . . . . . . 4

2.2 Basic properties . . . . . . . . . . . . . . . . 4

2.3 Examples .............................. 6

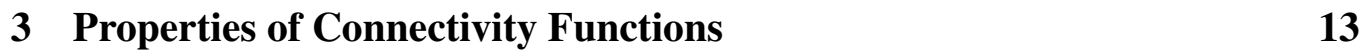

$3.1 \quad$ Connected components and direct sums . . . . . . . . . . . 13

3.2 Full closure . . . . . . . . . . . . . . . . . . . 15

3.3 Single-element removals $\ldots \ldots \ldots \ldots$

3.4 Single-element removals from matroids $\ldots \ldots \ldots$

3.5 The elision operation . . . . . . . . . . . . . . 21

3.6 Elision and extended connectivity functions . . . . . . . . . 22

4 Connectivity Functions on Small Sets 24

4.1 Connectivity functions on small sets . . . . . . . . . . . . 24

4.2 The elision operation and matroidal connectivity functions . . . . 32

5 Connectivity Functions and Polymatroids 36

5.1 Proof of the main theorem . . . . . . . . . . . . . 37

5.2 Connectivity polymatroids . . . . . . . . . . . . . . . 40

\begin{tabular}{ll}
\hline Bibliography & 43
\end{tabular} 


\section{Chapter 1}

\section{Introduction}

For a matroid $M$ on $E$, the connectivity function $\lambda_{M}$ is defined by $\lambda_{M}(A)=r(A)+$ $r(E-A)-r(M)$ for all $A \subseteq E$. The function $\lambda_{M}$ is symmetric and submodular and satisfies $\lambda_{M}(\emptyset)=0$. We say that any integer-valued set function with these properties is a connectivity function.

The connectivity function of a matroid captures many of the important properties. While connectivity functions are much more general than matroids, it turns out that many properties of matroids hold for connectivity functions in general. In particular properties associated with branch-width and tangles of a matroid tend to hold in this level of generality, see for example [1, 2]. Motivated by this, it is natural to study connectivity functions in their own right.

Cunningham [5, Section 8.2] conjectured that a connected matroid is determined, up to duality, by its connectivity function. Seymour [8] proved this conjecture is false in general by giving a counterexample. Let $E=\{1, \ldots 8\}$. Let $M$ be the rank 4 matroid on $E$ in which every 4 -set is a base except $\{1,2,3,4\},\{1,2,5,6\}$, and let every 4 -set except $\{1,2,3,4\},\{1,2,7,8\}$ be a base of $M^{\prime}$. Then $M$ and $M^{\prime}$ are connected, are not equal or dual, and yet have the same connectivity function $\lambda$ defined by

$$
\lambda(X)= \begin{cases}|X| & \text { if }|X| \leq 3 \\ |E-X| & \text { if }|X| \geq 5 \\ 3 & \text { if } X \in\{\{1,2,3,4\},\{5,6,7,8\},\{1,2,5,6\},\{3,4,7,8\}\} \\ 4 & \text { otherwise. }\end{cases}
$$

However, Cunningham's conjecture does hold in some special cases. The following theorem combines the results of Seymour [8] and Lemos [3, 4].

Theorem 1.1. Let $M$ and $N$ be connected matroids with the same ground set and the same connectivity function. If

(i) $M$ is binary, or 
(ii) $r(M) \neq r^{*}(M)$,

then $M=N$, or $M=N^{*}$.

The theorem tells us that the connectivity function of a matroid determines certain structure of a matroid, and even determine the matroid up to duality in some important special cases.

There are examples of connectivity functions that are not the connectivity functions of matroids. It is interesting to ask which connectivity functions come from a matroid, and if there are structures that give us all the connectivity functions. These questions also motivate our study of connectivity functions in general.

A polymatroid $P$ is an ordered pair $(E, r)$, where $E$ is a finite set and $r: 2^{E} \rightarrow \mathbb{Z}^{+} \cup$ $\{0\}$ is an integer-valued set function such that $r$ is increasing and submodular and satisfies $r(\emptyset)=0$. A fractional polymatroid is an ordered pair $(E, r)$, where $E$ is a finite set and $r: 2^{E} \rightarrow \mathbb{Q}^{+} \cup\{0\}$ is a set function that is increasing and submodular and satisfies $r(\emptyset)=0$. The fractional polymatroid on $E$ is half-integral if $r(A) \in$ $\left\{\frac{n}{2}: n \in \mathbb{Z}^{+} \cup\{0\}\right\}$ for all $A \subseteq E$.

The main theorem of this thesis is the following, which tells us that half-integral polymatroids give us all the connectivity functions.

Theorem 1.2. Let $\mu$ be a connectivity function on $E$. Then there is a half-integral polymatroid $P$ on $E$ such that $\mu=\lambda_{P}$.

We assume the reader has some basic knowledge of matroid theory, even though we will define the terminologies when we need to. The terminologies of matroid theory follow [5].

We now outline the structure of this thesis. In Chapter 2, we summarise some elementary but useful properties of connectivity functions and give examples of connectivity functions. This chapter will cover most of the preliminary knowledges.

In Chapter 3, we give more properties of connectivity functions and define the single-element removal from a connectivity function. In particular, we show that deletion and contraction of a matroid are just examples of single-element removal from the connectivity function of a matroid. We define a natural operation called elision to obtain a new unitary connectivity function by removing an element from an arbitrary unitary connectivity function in Section 3.5, and in Section 3.6 we discuss its relation between a well known function in matroid theory.

In Chapter 4, we find all the unitary connectivity function, up to isomorphism, with ground set of size less than 6 . We also give examples of unitary connectivity functions that are not from matroids and investigate the relation between these connectivity functions and elision operations. 
In Chapter 5, we prove our main result that every connectivity function is a connectivity function of a fractional polymatroid, and give results giving more specific details about the structure of polymatroids. 


\section{Chapter 2}

\section{Connectivity Functions}

In this chapter we first summarise some elementary properties of connectivity functions, and then give some examples of connectivity functions that arise naturally from different structures and review some background materials of these structures.

\subsection{Connectivity functions}

Let $E$ be a finite set. A set function on $E$ is a function whose domain is the power set of $E$, denoted $2^{E}$, and we call $E$ the ground set of the set function. An integervalued set function is a set function whose codomain is the set of integers. A set function $f$ on $E$ is normalised if $f(\emptyset)=0$; is symmetric if $f(X)=f(E-X)$ for all $X \subseteq E$; and is submodular if $f(X)+f(Y) \geq f(X \cup Y)+f(X \cap Y)$ for all $X, Y \subseteq E$. A normalised integer-valued set function $f$ on $E$ is unitary if $f(e) \leq 1$ for all $e \in E$.

An integer-valued set function $\mu: 2^{E} \rightarrow \mathbb{Z}$ is a connectivity function on $E$ if the following properties hold:

(C1) $\mu$ is normalised.

(C2) $\mu$ is symmetric.

(C3) $\mu$ is submodular.

\subsection{Basic properties}

This section summarises some elementary properties of connectivity functions.

Lemma 2.1. Let $\mu$ be a connectivity function on $E$, then $\mu(A) \geq 0$ for all $A \subseteq E$. 
Proof. By the symmetry and submodularity of $\mu$, we have

$$
\mu(A)=\frac{1}{2}(\mu(A)+\mu(E-A)) \geq \frac{1}{2}(\mu(\emptyset)+\mu(E))=\mu(\emptyset)=0 .
$$

Lemma 2.2. Let $\mu$ be a connectivity function on $E$. Let $X$ and $Y$ be disjoint subsets of $E$. Then $|\mu(X \cup Y)-\mu(X)| \leq \mu(Y)$.

Proof. The proof is by contradiction. Suppose $\mu(X \cup Y)-\mu(X)>\mu(Y)$. Then $\mu(X \cup Y)>\mu(X)+\mu(Y)$, contradicting the fact that $\mu$ is submodular. Suppose $\mu(X)-\mu(X \cup Y)>\mu(Y)$. Let $Z=E-(X \cup Y)$. Then by symmetry of $\mu$, we have $\mu(Y \cup Z)-\mu(Z)>\mu(Y)$, contradicting the submodularity of $\mu$.

We follow the convention in matroid theory to write $x$ instead of $\{x\}$ to denote the set containing the single element $x$.

The following corollaries are immediate consequences of Lemma 2.2 .

Corollary 2.3. Let $\mu$ be a connectivity function on $E$. Let $X \subseteq E, e \in E-X$. Then $|\mu(X \cup e)-\mu(X)| \leq \mu(e)$.

Corollary 2.4. Let $\mu$ be a unitary connectivity function on $E$. Let $X \subseteq E$, e $\in$ $E-X$. Then $|\mu(X \cup e)-\mu(X)| \leq 1$.

Lemma 2.5. Let $\mu$ be a connectivity function on $E$. Let $X \subseteq Y \subseteq E$ and $A \subseteq E-Y$. If $\mu(X \cup A)<\mu(X)$, then $\mu(Y \cup A)<\mu(Y)$. Moreover $\mu(X)-\mu(X \cup A) \leq \mu(Y)-$ $\mu(Y \cup A)$.

Proof. Suppose $\mu(Y) \leq \mu(Y \cup A)$. By adding $\mu(X)$ to both sides of the equation we have

$$
\mu(Y)+\mu(X) \leq \mu(Y \cup A)+\mu(X) .
$$

Since $\mu(X \cup A)<\mu(X)$, the equation above becomes

$$
\mu(Y)+\mu(X \cup A)<\mu(Y \cup A)+\mu(X),
$$

contradicting the submodularity of $\mu$.

Since $\mu$ is submodular, $\mu(Y)+\mu(X \cup A) \geq \mu(Y \cup A)+\mu(X)$. Rearranging the equation we have $\mu(X)-\mu(X \cup A) \leq \mu(Y)-\mu(Y \cup A)$.

Let $\mu$ be a connectivity function on $E$ and let $X, Y \subseteq E$. We say $X$ and $Y$ is a modular pair in $\mu$ if $\mu(X)+\mu(Y)=\mu(X \cup Y)+\mu(X \cap Y)$.

Lemma 2.6. Let $\mu$ be a unitary connectivity function on $E$. Let $X, Y \subseteq E$ be a modular pair in $\mu$ and let $e \in E$. If $\mu(X \cup e)<\mu(X)$ and $\mu(Y \cup e)<\mu(Y)$, then $\mu((X \cap Y) \cup e)<\mu(X \cap Y)$. 
Proof. $X$ and $Y$ is a modular pair in $\mu$, so $\mu(X)+\mu(Y)=\mu(X \cup Y)+\mu(X \cap Y)$. Since $\mu(X \cup e)<\mu(X)$, it follows from Corollary 2.4 that $\mu(X)=\mu(X \cup e)+1$. Similarly $\mu(Y \cup e)<\mu(Y)$ implies $\mu(Y)=\mu(Y \cup e)+1$. Notice that $e$ is not in both $X$ and $Y$, so $e \notin X \cup Y$. It follows from Lemma 2.5 that $\mu(X \cup Y)=$ $\mu(X \cup Y \cup e)+1$. Therefore the following holds from the substitutions of the equations above and the submodularity of $\mu$.

$$
\begin{aligned}
\mu(X \cap Y) & =\mu(X)+\mu(Y)-\mu(X \cup Y) \\
& =\mu(X \cup e)+\mu(Y \cup e)+2-\mu(X \cup Y) \\
& =\mu(X \cup e)+\mu(Y \cup e)+1-\mu(X \cup Y \cup e) \\
& \geq \mu(X \cup Y \cup e)+\mu((X \cap Y) \cup e)+1-\mu(X \cup Y \cup e) \\
& >\mu((X \cap Y) \cup e) .
\end{aligned}
$$

\subsection{Examples}

In this section, we give some examples of connectivity functions that arise naturally from different mathematical objects and some background materials of these objects which will be used later in this thesis.

\section{Connectivity functions of matroids}

The following concepts and results are well known and can be found in [5] but are presented in a somewhat different way there. Also any unexplained matroid terminologies that appear later in this thesis follow from [5].

A matroid $M$ is an ordered pair $(E, r)$, where $E$ is a finite set, and $r$ is an integervalued set function on $E$ satisfying the following properties:

(R1) $r$ is normalised.

(R2) $r(X) \leq|X|$, for all $X \subseteq E$.

(R3) $r(X) \leq r(Y)$, for all $X \subseteq Y \subseteq E$.

(R4) $r$ is submodular.

If $M$ is the matroid $(E, r)$, then $M$ is called the matroid on $E$ and the function $r$ is called the rank function of matroid $M$. We often denote the rank function of $M$ by $r_{M}$, in order to make it clear which matroid is being referred to. The rank of $M$, denoted $r(M)$, is defined to be $r(E)$. We refer to the property (R3) as the increasing property of $r$. Let $x \in E$. We say $x$ is a loop if $r(x)=0$ and call it a point if $r(x)=1$. Let $X \subseteq E$. We call $X$ a line if $r(X)=2$. 
Let $M$ be a matroid on $E$ and let $e \in E$. The deletion of $e$ from $M$, denoted $M \backslash e$, is a matroid on $E-e$, such that $r_{M \backslash e}(A)=r_{M}(E)$ for all $A \subseteq E-e$. The contraction of $e$ from $M$, denoted $M / e$, is a matroid on $E-e$, such that $r_{M / e}(A)=$ $r_{M}(A \cup e)-r_{M}(e)$ for all $A \subseteq E-e$. It is easy to verify that $M \backslash e$ and $M / e$ are both matroids. A matroid $N$ is a minor of $M$ if $N$ is obtained from $M$ by a sequence of deletion and contraction operations.

The connectivity function of a matroid $M$, denoted $\lambda_{M}$, is defined by

$$
\lambda_{M}(X)=r(X)+r(E-X)-r(M),
$$

for all $X \subseteq E$.

Lemma 2.7. Let $M$ be a matroid on $E$. Then the connectivity function $\lambda_{M}$ of $M$ is a unitary connectivity function.

Proof. It follows from (R1) that $r(\emptyset)=0$. Therefore

$$
\lambda_{M}(\emptyset)=r(\emptyset)+r(E)-r(M)=0+r(E)-r(M)=0 .
$$

Hence $\lambda_{M}$ is normalised, that is (C1) is satisfied. It follows directly from the definition of $\lambda_{M}$ that $\lambda_{M}$ is symmetric, so (C2) is also satisfied. It remains to prove that $(\mathrm{C} 3)$ is satisfied. Let $X$ and $Y$ be any subsets of $E$. An elementary fact called de Morgan's Law shows that $(E-X) \cup(E-Y)=E-(X \cap Y)$ and $(E-X) \cap(E-Y)=E-(X \cup Y)$. We use the submodularity of $r$ and de Morgan's Law to show $\lambda_{M}$ is submodualr,

$$
\begin{aligned}
& \lambda_{M}(X)+\lambda_{M}(Y) \\
= & r(X)+r(E-X)-r(M)+r(Y)+r(E-Y)-r(M) \\
\geq & r(X \cup Y)+r(X \cap Y)+r((E-X) \cup(E-Y)+r((E-X) \cap(E-Y))-2 r(M) \\
= & r(X \cup Y)+r(X \cap Y)+r(E-(X \cap Y))+r(E-(X \cup Y))-2 r(M) \\
= & \lambda_{M}(X \cup Y)+\lambda_{M}(X \cap Y) .
\end{aligned}
$$

Hence (C3) holds. Finally we show that $\lambda_{M}$ is unitary. It follows from (R2) that $r(x) \leq 1$ and from (R3) that $r(E-x) \leq r(E)$, for all $x \in E$, so

$$
\lambda_{M}(x)=r(x)+r(E-x)-r(M) \leq 1+r(E)-r(E) \leq 1 .
$$

Lemma 2.8. Let $r$ be a rank function of a matroid with ground set $E$ and $r^{*}$ be a function on $E$ defined as $r^{*}(X)=|X|+r(E-X)-r(E)$ for all $X \subseteq E$. Then $r^{*}$ is a rank function of a matroid.

Proof. It is trivial that $r^{*}(\emptyset)=0$, so (R1) holds. Since $r$ is a rank function, $r(E-$ $X)-r(E) \leq 0$ for all $X \subseteq E$. So it follows from the definition of $r^{*}$ that $r^{*}(X) \leq$ $|X|$, that is (R2) holds. Let $X, Y$ be subsets of $E$ such that $X \subseteq Y$. To show $r^{*}$ 
satisfies (R3), we only need to show $|X|+r(E-X) \leq|Y|+r(E-Y)$. It follows from the submodularity of $r$ that $r(E-X) \leq r(E-Y)+r(Y-X)$, that is $r(E-$ $X)-r(E-Y) \leq r(Y-X)$. Since $r$ satisfies (R2), then $r(Y-X) \leq|Y-X|=$ $|Y|-|X|$. Therefore $r(E-X)-r(E-Y) \leq|Y|-|X|$, rearraging the equation we have $|X|+r(E-X) \leq|Y|+r(E-Y)$. Let $A, B$ be subsets of $E$. To show $r^{*}$ satisfies (R4), we only need to show

$|A|+r(E-A)+|B|+r(E-B) \geq|A \cup B|+r(E-(A \cup B))+|A \cap B|+r(E-(A \cap B))$.

Since $|A|+|B|=|A \cup B|+|A \cap B|$, so we only need to show

$$
r(E-A)+r(E-B) \geq r(E-(A \cup B))+r(E-(A \cap B)) .
$$

By de Morgan's Law, $r(E-(A \cup B))=r((E-A) \cap(E-B))$ and $r(E-(A \cap B))=$ $r((E-A) \cup(E-B))$, so the equation above holds as $r$ is submodular.

The matroid in the last lemma, whose ground set is $E$ and whose rank function is $r^{*}$, is called the dual of $M$, denoted $M^{*}$. Thus $r_{M}^{*}=r_{M^{*}}$. It is easy to show that

$$
M / e=\left(M^{*} \backslash e\right)^{*} .
$$

The following lemma is a direct consequence of Lemma 2.8 .

Lemma 2.9. Let $M$ be a matroid on $E$. If $X \subseteq E$. Then

$$
\lambda_{M}(X)=r(X)+r^{*}(X)-|X| \text {. }
$$

The following corollary is an immediate consequence of Lemma 2.9

Corollary 2.10. Let $M$ be a matroid. Then $\lambda_{M}=\lambda_{M^{*}}$.

It is not true that every unitary connectivity function is a connectivity function of a matroid. We want to know what structures forbid the matroids to have these connectivity functions. Examples and discussions of these connectivity functions are given in Chapter 4 .

\section{Connectivity functions of graphs}

A graph $G=(V, E)$ consists of a set $V$ of vertices and a set $E$ of edges such that for all $e \in E$, there exist $u, v \in V$ such that $e=\{u, v\}$. The definition is saying every edge is a 2-element multiset of $V$, for convenience, edge $e=\{u, v\}$ is denoted by $u v$, and vertices $u$ and $v$ are said to be incident with edge $e$, and vice versa. We also call $u$ and $v$ the ends of edge $e=\{u, v\}$ and say $u$ is adjacent to $v$.

In order to have a good intuition of a graph, we usually graphically represent a graph. Each vertex is indicated by a point, and each edge is indicated by a line joining the points which represent its ends. Here is an example of representing a graph. 


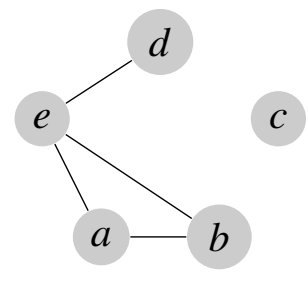

Figure 2.1: Graphical representation of graph $G$.

Example 2.11. Let $G=(E, V)$ be a graph, where $V=\{a, b, c, d, e\}$ and $E=$ $\{\{a, b\},\{a, e\},\{b, e\},\{d, e\}\}$. Figure above is a representation of $G$.

Let $G=(V, E)$ be a graph and let $X \subseteq E$. We use $V(X)$ to denote the set of vertices that are incident with at least one edge in $X$. The graph connectivity function $\lambda_{G}$ is defined as

$$
\lambda_{G}(X)=|V(X)|+|V(E-X)|-|V(E)|,
$$

for all $X \subseteq E$. It is not hard to see $\lambda_{G}(X)$ measures the number of vertices that are both incident with $X$ and $E-X$.

Lemma 2.12. Let $G=(V, E)$ be a graph. Let $X$ and $Y$ be subsets of $E$. Then

$$
|V(X)|+|V(Y)| \geq|V(X \cup Y)|+|V(X \cap Y)| .
$$

Proof. It follows from the basic fact, called the principle of inclusion-exclusion, in set theory that $|V(X)|+|V(Y)|=|V(X) \cup V(Y)|+|V(X) \cap V(Y)|$. Also it is easy to see that $V(X) \cup V(Y)=V(X \cup Y)$ and $V(X) \cap V(Y) \supseteq V(X \cap Y)$. So $|V(X)|+$ $|V(Y)| \geq|V(X \cup Y)|+|V(X \cap Y)|$.

Lemma 2.13. Let $G=(V, E)$ be a graph. Then the connectivity function $\lambda_{G}$ of $G$ is a connectivity function.

Proof. It is trivial that $\lambda_{G}(\emptyset)=0$, hence (C1) holds. It follows from the definition of $v$ that $\lambda_{G}$ is symmetric, hence (C2) holds. We only need to show $\lambda_{G}$ is submodular. Let $X$ and $Y$ be two subsets of $E$. It follows from the definition of $\lambda_{G}$, to show $\lambda_{G}(X)+\lambda_{G}(Y) \geq \lambda_{G}(X \cup Y)+\lambda_{G}(X \cap Y)$ we only need to show

$$
\begin{aligned}
& |V(X)|+|V(E-X)|+|V(Y)|+|V(E-Y)| \\
\geq & |V(X \cup Y)|+|V(E-(X \cup Y))|+|V(X \cap Y)|+|V(E-(X \cap Y))| .
\end{aligned}
$$

By Lemma 2.12, we have

$$
|V(X)|+|V(Y)| \geq|V(X \cup Y)|+|V(X \cap Y)|,
$$

and

$$
\begin{aligned}
|V(E-X)|+|V(E-Y)| & \geq|V((E-X) \cup(E-Y))|+|V((E-X) \cap(E-Y))| \\
& =|V(E-(X \cap Y))|+|V(E-(X \cup Y))| .
\end{aligned}
$$

Combining (2.3) and (2.4), we can see (2.2) holds, hence $\lambda_{G}$ is submodular. Therefore $(\mathrm{C} 3)$ holds. 
Notice that the connectivity function of a graph is not unitary in general.

The following example will generalise the examples in matroids and graphs.

\section{Connectivity functions of polymatroids}

A polymatroid $P$ is an ordered pair $(E, r)$, where $E$ is a finite set, and $r$ is a integervalued set function on $E$ satisfying the following properties:

(P1) $r$ is normalised.

(P2) $r$ is increasing.

(P3) $r$ is submodular.

If $P$ is the polymatroid $(E, r)$, then $P$ is called the polymatroid on $E$ and the function $r$ is called the rank function of $P$. We often denote the rank function of $P$ by $r_{P}$. The rank of $P$, denoted $r(P)$, is defined to be $r(E)$. The polymatroid $P$ is a $k$-polymatroid if $r(\{x\}) \leq k$ for all $x \in E$, where $k$ is a positive integer. We can see that every matroid is just a 1-polymatroid.

Let $G=(V, E)$ be a graph. Define the set function $r_{G}$ on $E$ to be

$$
r_{G}(X)=|V(X)|
$$

for all $X \subseteq E$. Then it is not hard to see $P=\left(E, r_{G}\right)$ is a 2-polymatroid.

Lemma 2.14. Let $G=(V, E)$ be a graph. Let $r_{G}$ be a function defined as above. Then $P=\left(E, r_{G}\right)$ is a 2-polymatroid.

Proof. It is trivial that (P1) holds. Let $X$ and $Y$ be subsets of $E$, then $|V(X)| \leq$ $|V(Y)|$, so $r_{G}(X) \leq r_{G}(Y)$. Hence (P2) holds. Lemma 2.12 shows (P3) holds. Also $r(e) \leq 2$ for all $e \in E$. So $P$ is a 2-polymatroid.

Geometrically, a 2-polymatroid can be thought of as a multiset of lines, points, and loops of some matroid [7, Section 2].

The connectivity function of a polymatroid $P$, denoted $\lambda_{P}$, is defined as

$$
\lambda_{P}(X)=r(X)+r(E-X)-r(P),
$$

for all $X \subseteq E$.

Lemma 2.15. Let $P=(E, r)$ be a polymatroid. Then the connectivity function $\lambda_{P}$ of $P$ is a connectivity function.

Proof. In the proof of Lemma 2.7, we only use (R1) that $r$ is normalised, (R4) that $r$ is submodular and de Morgan's Law. As a polymatroid is just a matroid except that we drop the condition (R2), the proof of Lemma 2.7] is also valid for this lemma, except that $\lambda_{P}$ is not unitary. 


\section{The extended connectivity $\kappa$ in matroid}

Let $M$ be a matroid on $E$ and let $\lambda_{M}$ be the connectivity function of $M$. Let $X$ and $Y$ be two disjoint sets of $E$. The extended connectivity of $X$ and $Y$, denoted $\kappa_{M}(X, Y)$, is defined as

$$
\kappa_{M}(X, Y)=\min \left\{\lambda_{M}(X \cup Z): Z \subseteq E-(X \cup Y)\right\}
$$

It is not hard to see that $\kappa_{M}(Y, X)=\kappa_{M}(X, Y)$. Moreover,

$$
\kappa_{M}(X, E-X)=\lambda_{M}(X) .
$$

The extended connectivity $\kappa$ is well known in some area of matroid theory. For example Tutte's Linking Theorem which generalizes Menger's Theorem is a result about $\kappa$. The reader can find more detail in [5, Section 8.5].

Now we define a connectivity function which is based on $\kappa$. Let $M$ be a matroid on $E$ and let $A \subseteq E$. Define the function $\kappa_{\left(\lambda_{M}, A\right)}$ on $A$ by

$$
\kappa_{\left(\lambda_{M}, A\right)}(X)=\kappa_{M}(X, A-X)
$$

for all $X \subseteq A$. The following lemma shows this is a connectivity function.

Lemma 2.16. Let $M$ be a matroid on $E$ and let $A \subseteq E$. Then $\kappa_{\left(\lambda_{M}, A\right)}$ is a unitary connectivity function on A.

Proof. It is trivial that (C1) holds. It follows from the definition of $\kappa_{\left(\lambda_{M}, A\right)}$ and $\kappa$ that $\kappa_{\left(\lambda_{M}, A\right)}$ is symmetric. We only need to show $\kappa_{\left(\lambda_{M}, A\right)}$ is submodular. Let $X$ and $Y$ be subsets of $A$. Let $B$ and $C$ be subsets of $E-A$ such that $\kappa_{\left(\lambda_{M}, A\right)}(X)=$ $\lambda_{M}(X \cup B)$ and $\kappa_{\left(\lambda_{M}, A\right)}(Y)=\lambda(Y \cup C)$. By the submodularity of $\lambda_{M}$, we have

$$
\lambda_{M}(X \cup B)+\lambda_{M}(Y \cup C) \geq \lambda_{M}(X \cup Y \cup B \cup C)+\lambda_{M}((X \cup B) \cap(Y \cup C)) .
$$

By the definition of $\kappa_{\left(\lambda_{M}, A\right)}$, Equation $(2.5)$ becomes

$$
\kappa_{\left(\lambda_{M}, A\right)}(X)+\kappa_{\left(\lambda_{M}, A\right)}(Y) \geq \lambda_{M}(X \cup Y \cup B \cup C)+\lambda_{M}((X \cup B) \cap(Y \cup C)) .
$$

It follows from the definition of $\kappa_{\left(\lambda_{M}, A\right)}$ and $\kappa_{M}$ that

$$
\kappa_{\left(\lambda_{M}, A\right)}(X \cup Y)=\kappa_{M}(X \cup Y, A-(X \cup Y)) \leq \lambda_{M}((X \cup Y) \cup(B \cup C)),
$$

and

$$
\kappa_{\left(\lambda_{M}, A\right)}(X \cap Y)=\kappa_{M}(X \cap Y, A-(X \cap Y)) \leq \lambda_{M}((X \cap Y) \cup(B \cap C)) .
$$

Comparing (2.6) with 2.7) and (2.8), we can see $\kappa_{\left(\lambda_{M}, A\right)}$ is submodular. Since $\lambda_{M}$ is unitary, it is trivial that $\kappa_{\left(\lambda_{M}, A\right)}$ is also unitary. 
If we read carefully in the proof of Lemma 2.16, it is easy to see we use nothing more than the fact that $\lambda_{M}$ is a connectivity function. So this construction will work for all connectivity functions.

We now generalise the concept and the lemma. Let $\mu$ be a connectivity function on $E$ and let $X$ and $Y$ be two disjoint subsets of $E$. Then the extended connectivity of $X$ and $Y$ in $\mu$, denoted $\kappa_{\mu}(X, Y)$, is defined as

$$
\kappa_{\mu}(X, Y)=\min \{\mu(X \cup Z): Z \subseteq E-(X \cup Y)\} .
$$

Let $A \subseteq E$. Define the function $\kappa_{(\mu, A)}(X)$ on $A$ by

$$
\kappa_{(\mu, A)}(X)=\kappa_{\mu}(X, A-X)
$$

for all $X \subseteq A$.

Lemma 2.17. Let $\mu$ be a connectivity function on $E$ and let $A \subseteq E$. Then $\kappa_{(\mu, A)}$ is a connectivity function on $A$. Moreover, if $\mu$ is unitary, then $\kappa_{(\mu, A)}$ is also unitary.

Surprisingly we show that this way of constructing unitary connectivity functions coincides with a natural operation in unitary connectivity functions later in Chapter 3 . 


\section{Chapter 3}

\section{Properties of Connectivity Functions}

In this chapter we give some properties of connectivity functions. Later on we study ways to remove elements from the ground set of connectivity functions. But first we study direct sums of connectivity functions which agrees with the notion of direct sums of matroids.

\subsection{Connected components and direct sums}

We define a connectivity function $\mu$ on $E$ to be connected if $\mu(A) \neq 0$ for all nonempty proper subsets $A$ of $E$, otherwise $\mu$ is disconnected. The following lemma shows how a connectivity function can be naturally broken up into smaller ones.

Lemma 3.1. Let $\mu$ be a connectivity function on $E$, and let $(A, B)$ be a partition of $E$, such that $\mu(A)=0$. Define $\mu^{\prime}$ on $A$ by $\mu^{\prime}(X)=\mu(X)$ for all $X \subseteq A$. Then $\mu^{\prime}$ is a connectivity function on $A$.

Proof. We first show that $\mu^{\prime}$ is submodular. Let $X, Y \subseteq A$. Then $X \cup Y$ and $X \cap Y$ are both subsets of $A$. So it follows from the submodularity of $\mu$ that

$\mu^{\prime}(X)+\mu^{\prime}(Y)=\mu(X)+\mu(Y) \geq \mu(X \cap Y)+\mu(X \cup Y)=\mu^{\prime}(X \cap Y)+\mu^{\prime}(X \cup Y)$.

We next show that $\mu^{\prime}$ is symmetric. It follows from the symmetry of $\mu$ that, $\mu^{\prime}(X)=\mu(X)=\mu(E-X)=\mu(B \cup(A-X)$. So it follows from the submodularity of $\mu$ that

$$
\mu(B)+\mu(A-X) \geq \mu(B \cup(A-X))=\mu^{\prime}(X) .
$$

Notice that $\mu(B)=\mu(A)=0$, so we have $\mu^{\prime}(A-X) \geq \mu^{\prime}(X)$. On the other hand, it follows from the submodularity of $\mu$ that

$$
\mu(A)+\mu(B \cup(A-X)) \geq \mu(E)+\mu(A-X) .
$$


Notice that $\mu(A)=\mu(E)=0$, and $\mu(X)=\mu(B \cup(A-X))$. So 3.1 becomes $\mu^{\prime}(X) \geq \mu^{\prime}(A-X)$. Hence $\mu^{\prime}(X)=\mu^{\prime}(A-X)$. Therefore $\mu^{\prime}$ is a connectivity function on $A$.

Let $\mu$ be a connectivity function on $E$ and let $A \subseteq E$. We say $A$ is a separator of $\mu$ if $\mu(A)=0$, and $A$ is a component of $\mu$ if it is a minimal nonempty separator.

We can also construct a connectivity function from two given connectivity functions. Let $\mu_{1}$ and $\mu_{2}$ be two connectivity functions on the sets $E_{1}$ and $E_{2}$ respectively. The direct sum of connectivity functions $\mu_{1}$ and $\mu_{2}$, denoted $\mu_{1} \oplus \mu_{2}$, is defined as $\mu_{1} \oplus \mu_{2}(A)=\mu_{1}\left(A \cap E_{1}\right)+\mu_{2}\left(A \cap E_{2}\right)$ for all $A \subseteq E_{1} \cup E_{2}$. We need to show the direct sum is indeed a connectivity function.

Lemma 3.2. Let $\mu_{1}$ and $\mu_{2}$ be connectivity functions on the set $E_{1}$ and $E_{2}$ respectively, where $E_{1} \cap E_{2}=\emptyset$. Then $\mu_{1} \oplus \mu_{2}$ is a connectivity function on $E_{1} \cup E_{2}$. Moreover $\mu\left(E_{1}\right)=\mu\left(E_{2}\right)=0$.

Proof. It is trivial that $\mu_{1} \oplus \mu_{2}$ is normalised. Let $A, B \subseteq E_{1} \cup E_{2}$. We first show $\mu_{1} \oplus \mu_{2}$ is symmetric. It follows from the symmetry of $\mu_{1}$ and $\mu_{2}$ that $\mu_{1}\left(A \cap E_{1}\right)=$ $\mu_{1}\left((E-A) \cap E_{1}\right)$ and $\mu_{2}\left(A \cap E_{2}\right)=\mu_{2}\left((E-A) \cap E_{2}\right)$. So

$$
\begin{aligned}
\mu_{1} \oplus \mu_{2}(A) & =\mu_{1}\left(A \cap E_{1}\right)+\mu_{2}\left(A \cap E_{2}\right) \\
& =\mu_{1}\left((E-A) \cap E_{1}\right)+\mu_{2}\left((E-A) \cap E_{2}\right) \\
& =\mu_{1} \oplus \mu_{2}(E-A) .
\end{aligned}
$$

Next we show $\mu_{1} \oplus \mu_{2}$ is submodular. The following argument follows from the definition of direct sums, the submodularity of $\mu_{1}$ and $\mu_{2}$, and de Morgan's Law.

$$
\begin{aligned}
& \mu_{1} \oplus \mu_{2}(A)+\mu_{1} \oplus \mu_{2}(B) \\
= & \mu_{1}\left(A \cap E_{1}\right)+\mu_{2}\left(A \cap E_{2}\right)+\mu_{1}\left(B \cap E_{1}\right)+\mu_{2}\left(B \cap E_{2}\right) \\
\geq & \mu_{1}\left(\left(A \cap E_{1}\right) \cap\left(B \cap E_{1}\right)\right)+\mu_{1}\left(\left(A \cap E_{1}\right) \cup\left(B \cap E_{1}\right)\right) \\
& +\mu_{2}\left(\left(A \cap E_{2}\right) \cap\left(B \cap E_{2}\right)\right)+\mu_{2}\left(\left(A \cap E_{2}\right) \cup\left(B \cap E_{2}\right)\right) \\
= & \mu_{1}\left((A \cap B) \cap E_{1}\right)+\mu_{1}\left((A \cup B) \cap E_{1}\right)+\mu_{2}\left((A \cap B) \cap E_{2}\right)+\mu_{2}\left((A \cup B) \cap E_{2}\right) \\
= & \mu_{1} \oplus \mu_{2}(A \cap B)+\mu_{1} \oplus \mu_{2}(A \cup B) .
\end{aligned}
$$

Therefore $\mu_{1} \oplus \mu_{2}$ is a connectivity function. In particular, it follows from the definition of $\mu_{1} \oplus \mu_{2}$ that

$$
\mu_{1} \oplus \mu_{2}\left(E_{1}\right)=\mu_{1}\left(E_{1} \cap E_{1}\right)+\mu_{2}\left(E_{1} \cap E_{2}\right)=\mu_{1}\left(E_{1}\right)+\mu_{2}(\emptyset)=0,
$$

and $\mu_{1} \oplus \mu_{2}\left(E_{2}\right)=\mu_{1} \oplus \mu_{2}\left(E_{1}\right)=0$ as $\mu_{1} \oplus \mu_{2}$ is symmetric.

Let $M$ be a matroid on $E$. A separator of $M$ is a subset $X$ such at that $\lambda_{M}(X)=0$, and $X$ is a component of $M$ if it is a minimal nonempty separator. A matroid is connected if the only separators of $M$ are $\emptyset$ and $E$. 
It is not hard to see the notion of connectedness of matroids agrees with the connectedness of connectivity function, and the following lemma holds.

Lemma 3.3. Let $M$ be a matroid on $E$. Then $X \subseteq E$ is a component of $M$ if and only if $X$ is a component of $\lambda_{M}$, where $\lambda_{M}$ is the connectivity function of $M$.

From Lemma 3.1 and Lemma 3.2, we know that every disconnected connectivity system is just the direct sum of its connected components, so we will only focus on connected connectivity functions in the rest of this thesis.

\subsection{Full closure}

As we know there are many equivalent ways to define a matroid [5], such as independent sets, rank functions, closure operators, bases, etc. We would like to know if there is another way to define connectivity functions, as presenting a connectivity function with the value on each subset of the ground set is very inconvenient when the size of the ground set is large. In this section we try to define a connectivity function in term of a closure operator called full closure and we show that full closure cannot reconstruct a connectivity function.

Let $\mu$ be a connectivity function on $E$. A set $A \subseteq E$ is fully-closed if $\mu(A \cup e)>$ $\mu(A)$ for all $e \in E-A$. The full closure of $A$, denoted $\operatorname{fcl}_{\mu}(A)$ or $\operatorname{fcl}(A)$ if $\mu$ is clear from the context, is the intersection of all fully-closed sets that contains $A$. It follows from the next lemma and the definition of fully-closed set and full closure, if $\mu(A \cup e) \leq \mu(A)$ then $e \in \operatorname{fcl}_{\mu}(A)$.

We will show $\mathrm{fcl}(X)$ is fully-closed. We begin with a preliminary lemma.

Lemma 3.4. Let $\mu$ be a connectivity function on $E$ and let $A, B$ be subsets of $E$ such that $A \subseteq B$. If $\mu(A) \geq \mu(A \cup e)$, then $\mu(B) \geq \mu(B \cup e)$.

Proof. If $\mu(A) \geq \mu(A \cup e)$, then by the symmetry of $\mu$ we have $\mu(E-A) \geq \mu(E-$ $(A \cup e))$. Since $A \subseteq B$, we have $E-(B \cup e) \subseteq E-(A \cup e)$. By the contrapositive of Lemma 2.5, we have $\mu(E-B) \geq \mu(E-(B \cup e))$. So the result follows from the symmetry of $\mu$.

Lemma 3.5. Let $\mu$ be a connectivity function on $E$. Then $\mathrm{fcl}(X)$ is fully-closed for all $X \subseteq E$.

Proof. We show the intersection of two fully-closed sets is fully-closed, which will suffice to show the lemma. Let $A, B \subseteq E$ be two fully-closed sets. Suppose $A \cap B$ is not fully-closed. Then there exists $e \in E-(A \cap B)$ such that $\mu(A \cap B) \geq$ $\mu((A \cap B) \cup e)$. By Lemma 3.4, we have $\mu(A) \geq \mu(A \cup e)$, contradicting the fact that $A$ is fully-closed. Thus $A \cap B$ is fully-closed. 
A closure operator on a set $E$ is a function $\mathrm{cl}: 2^{E} \rightarrow 2^{E}$ satisfying the following properties.

(i) $X \subseteq \operatorname{cl}(X)$, for all $X \subseteq E$.

(ii) $\operatorname{cl}(\operatorname{cl}(X))=\operatorname{cl}(X)$, for all $X \subseteq E$.

(iii) If $X \subseteq Y \subseteq E$, then $\operatorname{cl}(X) \subseteq \operatorname{cl}(Y)$.

We next show full closure is a closure operator.

Lemma 3.6. Let $\mu$ be a unitary connectivity function on $E$. Then $\mathrm{fcl}$ is a closure operator.

Proof. It follows from the definition of full closure $X \subseteq \mathrm{fcl}(X)$. By definition, $\mathrm{fcl}(\mathrm{fcl}(X))$ is the intersection of all fully-closed sets containing $\mathrm{fcl}(X)$, we have $\mathrm{fcl}(\mathrm{fcl}(X)) \supseteq \mathrm{fcl}(X)$. By Lemma 3.5. we know $\mathrm{fcl}(X)$ is closed and contains $\mathrm{fcl}(X)$, so $\mathrm{fcl}(\mathrm{fcl}(X)) \subseteq \mathrm{fcl}(X)$. Therefore $\mathrm{fcl}(\mathrm{fcl}(X))=\mathrm{fcl}(X)$. Suppose $X \subseteq$ $Y \subseteq E$. Let $P$ be the intersection of all fully-closed sets containing $X$ but not containing $Y$ and $Q$ be the intersection of all fully-closed sets containing $X$ and $Y$. Then $\mathrm{fcl}(X)=P \cap Q$ and $\mathrm{fcl}(Y)=Q$, thus $\mathrm{fcl}(X) \subseteq \mathrm{fcl}(Y)$.

Unfortunately, the collection of fully-closed sets of a connectivity function on a set cannot uniquely determine the connectivity function. For example the collection of fully-closed sets in $K_{4,1}$ and $K_{4,2}$, which are shown in Table 4.1 , are both $\{\emptyset, E\}$.

\subsection{Single-element removals}

We have defined the minor of a matroid in Section 2.3. We would like to generalise the similar notion of minor for a connectivity function, that is, we want to define operations that produce a new connectivity function from a given connectivity function by removing an element but still preserve some structure of the original connectivity function.

The following lemma describes what properties the new connectivity function will have. Later in Section 3.5, we will define a very natural operation that generalises the similar notion of the minor of a unitary connectivity function.

Lemma 3.7. Let $\mu$ be a unitary connectivity function on $E$ and $e \in E$. For all $A, X, Y \subseteq E-e$, if $\mu^{\prime}$ satisfies the following properties, then $\mu^{\prime}$ is a unitary connectivity function.

(i) $\mu^{\prime}(A) \in\{\mu(A), \mu(A)-1\}$.

(ii) If $\mu(A)=\mu(A \cup e)$, then $\mu^{\prime}(A)=\mu^{\prime}(E-(A \cup e))$. 
(iii) If $\mu(A) \neq \mu(A \cup e)$, then $\mu^{\prime}(A)=\min \{\mu(A), \mu(A \cup e)\}$.

(iv) Let $A \subseteq A^{\prime} \subseteq E-\{$ e $\}$. If $\mu^{\prime}(A)=\mu(A)-1$, then $\mu^{\prime}\left(A^{\prime}\right)=\mu\left(A^{\prime}\right)-1$.

(v) If $\mu^{\prime}(X)=\mu(X)-1, \mu^{\prime}(Y)=\mu(Y)-1$, and $\mu(X)+\mu(Y)=\mu(X \cup Y)+$ $\mu(X \cap Y)$, then $\mu^{\prime}(X \cap Y)=\mu(X \cap Y)-1$.

Proof. We first show that $\mu^{\prime}$ is symmetric. Let $\{A, e, B\}$ be a partition of $E$. We need to show $\mu^{\prime}(A)=\mu^{\prime}(B)$. If $\mu(A)=\mu(A \cup e)$, then by property (ii), $\mu^{\prime}(A)=$ $\mu^{\prime}(E-(A \cup e))=\mu^{\prime}(B)$. If $\mu(A) \neq \mu(A \cup e)$, then by the symmetry of $\mu$, we have $\mu(B \cup e) \neq \mu(B)$. Hence by property (iii),

$$
\mu^{\prime}(A)=\min \{\mu(A), \mu(A \cup e)\}=\min \{\mu(B \cup e), \mu(B)\}=\mu^{\prime}(B) .
$$

We next show $\mu^{\prime}$ is submodular by considering different cases. Notice that property (i) implies $\mu(A) \geq \mu^{\prime}(A)$ for all $A \subseteq E-e$. Let $X, Y \subseteq E$.

Suppose $\mu^{\prime}(X)=\mu(X)$ and $\mu^{\prime}(Y)=\mu(Y)$, then by the submodularity of $\mu$ and (i), obtain

$\mu^{\prime}(X)+\mu^{\prime}(Y)=\mu(X)+\mu(Y) \geq \mu(X \cup Y)+\mu(X \cap Y) \geq \mu^{\prime}(X \cup Y)+\mu^{\prime}(X \cap Y)$.

Suppose $\mu^{\prime}(X)=\mu(X)$ and $\mu^{\prime}(Y)=\mu(Y)-1$. Then by submodularity of $\mu$, obtain

$$
\mu^{\prime}(X)+\mu^{\prime}(Y)=\mu(X)+\mu(Y)-1 \geq \mu(X \cup Y)+\mu(X \cap Y)-1 .
$$

And it follows from property (iv) that $\mu(X \cup Y)=\mu^{\prime}(X \cup Y)+1$, and it follows from property (i) that $\mu(X \cap Y) \geq \mu^{\prime}(X \cap Y)$. Hence

$$
\mu^{\prime}(X)+\mu^{\prime}(Y) \geq \mu(X \cup Y)+\mu(X \cap Y)-1 \geq \mu^{\prime}(X \cup Y)+\mu^{\prime}(X \cap Y) .
$$

Suppose $\mu^{\prime}(X)=\mu(X)-1$ and $\mu^{\prime}(Y)=\mu(Y)-1$. By submodularity of $\mu$, obtain

$$
\mu^{\prime}(X)+\mu^{\prime}(Y)=\mu(X)+\mu(Y)-2 \geq \mu(X \cup Y)+\mu(X \cap Y)-2 .
$$

If $X$ and $Y$ is not a modular pair in $\mu$, then $(3.3)$ becomes

$$
\mu^{\prime}(X)+\mu^{\prime}(Y)=\mu(X)+\mu(Y)-2>\mu(X \cup Y)+\mu(X \cap Y)-2 .
$$

But (3.4) is just the same as 3.2). Hence $\mu^{\prime}$ is submodular.

If $X$ and $Y$ is a modular pair in $\mu$, then 3.3 becomes

$$
\mu^{\prime}(X)+\mu^{\prime}(Y)=\mu(X)+\mu(Y)-2=\mu(X \cup Y)+\mu(X \cap Y)-2 .
$$

It follows from property (iv) that $\mu^{\prime}(X \cup Y)=\mu(X \cup Y)-1$, and from (v) that $\mu^{\prime}(X \cap Y)=\mu(X \cap Y)-1$. Then 3.5 becomes

$$
\mu^{\prime}(X)+\mu^{\prime}(Y)=\mu(X \cup Y)+\mu(X \cap Y)-2=\mu^{\prime}(X \cup Y)+\mu^{\prime}(X \cap Y) .
$$

Therefore $\mu^{\prime}$ is submodular. 
We say the connectivity function $\mu^{\prime}$ is a single-element removal of $e$ from $\mu$ if $\mu^{\prime}$ satisfies all the properties in Lemma 3.7 .

We introduce two examples of single-element removal that arise naturally from connectivity function of matroids in the next section.

\subsection{Single-element removals from matroids}

In this section we show that deletion or contraction of an element in a matroid just corresponds to a single-element removal from the connectivity function of the matroid.

Let $M$ be a matroid with ground set $E$ and let $e \in E$. Recall that the deletion of $e$ from $M$, denoted $M \backslash e$, is a matroid on $E-e$ such that $r_{M \backslash e}(A)=r_{M}(E)$ for all $A \subseteq E-e$, and the contraction of $e$ from $M$, denoted $M / e$, is a matroid on $E-e$ such that $r_{M / e}(A)=r_{M}(A \cup e)-r_{M}(e)$ for all $A \subseteq E-e$. Let $M^{*}$ be the dual of $M$. It is easy to show that $M / e=\left(M^{*} \backslash e\right)^{*}$. The closure of $X \subseteq E$, denoted $\mathrm{cl}_{M}$, is defined by

$$
\operatorname{cl}_{M}(X)=\left\{x \in E: r_{M}(X \cup x)=r_{M}(X)\right\} .
$$

The following lemma gives formulas for the connectivity functions of a matroid by deleting and contracting a element from a given matroid.

Lemma 3.8. Let $M$ be a matroid with ground set $E$, and let $A \subseteq E$. Then

$$
\lambda_{M \backslash e}(A)= \begin{cases}\lambda_{M}(A)-1 & \text { if } e \in \mathrm{cl}_{M^{*}}(A) \\ \lambda_{M}(A) & \text { else, }\end{cases}
$$

and

$$
\lambda_{M / e}(A)= \begin{cases}\lambda_{M}(A)-1 & \text { if } e \in \mathrm{cl}_{M}(A) \\ \lambda_{M}(A) & \text { else. }\end{cases}
$$

Proof. By definition of the connectivity function of a matroid, we have

$$
\begin{aligned}
& \lambda_{M \backslash e}(A)=r_{M \backslash e}(A)+r_{(M \backslash e)^{*}}(A)-|A|=r_{M \backslash e}(A)+r_{M^{*} / e}(A)-|A| . \\
& \lambda_{M / e}(A)=r_{M / e}(A)+r_{(M / e)^{*}}(A)-|A|=r_{M / e}(A)+r_{M^{*} \backslash e}(A)-|A| .
\end{aligned}
$$

Notice that $r_{M \backslash e}(A)=r_{M}(A)$ and $r_{M^{*} \backslash e}(A)=r_{M^{*}}(A)$ we can simplify the two equations above,

$$
\begin{gathered}
\lambda_{M \backslash e}(A)=\lambda_{M}(A)+r_{M^{*} / e}(A)-r_{M^{*}}(A) \\
\lambda_{M / e}(A)=\lambda_{M}(A)+r_{M / e}(A)-r_{M}(A) .
\end{gathered}
$$

We can see that $\lambda_{M \backslash e}(A)=\lambda_{M}(A)-1$ only when $r_{M^{*}}(A)>r_{M^{*} / e}(A)$ that is $e \in$ $\mathrm{cl}_{M^{*}}(A)$. Rewrite equation 3.9 .

$$
\lambda_{M \backslash e}(A)= \begin{cases}\lambda_{M}(A)-1 & \text { if } e \in \operatorname{cl}_{M^{*}}(A) \\ \lambda_{M}(A) & \text { else. }\end{cases}
$$


Similarly, equation 3.10 becomes

$$
\lambda_{M / e}(A)= \begin{cases}\lambda_{M}(A)-1 & \text { if } e \in \mathrm{cl}_{M}(A) \\ \lambda_{M}(A) & \text { else. }\end{cases}
$$

The next lemma shows the connectivity function of matroids by deleting and contracting an element from a matroid satisfies property (iv) in Lemma 3.7 of being a single-element removal of the connectivity of the given matroid.

Lemma 3.9. Let $M$ be a matroid with ground set $E$ and let $A \subseteq A^{\prime} \subseteq E-e$.

(i) If $\lambda_{M \backslash e}(A)=\lambda_{M}(A)-1$, then $\lambda_{M \backslash e}\left(A^{\prime}\right)=\lambda_{M}\left(A^{\prime}\right)-1$.

(ii) If $\lambda_{M / e}(A)=\lambda_{M}(A)-1$, then $\lambda_{M / e}\left(A^{\prime}\right)=\lambda_{M}\left(A^{\prime}\right)-1$.

Proof. If $\lambda_{M \backslash e}(A)=\lambda_{M}(A)-1$, then by Lemma 3.8, $e \in \mathrm{cl}_{M^{*}}(A)$, which implies $e \in \mathrm{cl}_{M^{*}}\left(A^{\prime}\right)$. Hence by Lemma 3.8, we have $\lambda_{M \backslash e}\left(A^{\prime}\right)=\lambda_{M}\left(A^{\prime}\right)-1$. If $\lambda_{M / e}(A)=$ $\lambda_{M}(A)-1$ then by Lemma $3.8 e \in \operatorname{cl}_{M}(A)$, which implies $e \in \operatorname{cl}_{M}\left(A^{\prime}\right)$. Hence by Lemma 3.8, we obtain $\lambda_{M / e}\left(A^{\prime}\right)=\lambda_{M}\left(A^{\prime}\right)-1$.

Let $M$ be a matroid on $E$ and let $X, Y \subseteq E$. We say $X$ and $Y$ is a modular pair in $M$ if $r_{M}(X)+r_{M}(Y)=r_{M}(X \cup Y)+r_{M}(X \cap Y)$. Let $\mu$ be a connectivity function on $E$ and $X, Y \subseteq E$. Recall that, $X$ and $Y$ is a modular pair in $\mu$ if $\mu(X)+\mu(Y)=$ $\mu(X \cup Y)+\mu(X \cap Y)$.

The following lemma shows the relation between modular pairs in matroid and modular pairs in its connectivity function.

Lemma 3.10. Let $M$ be a matroid on $E$ and let $X, Y \subseteq E$. Then $X$ and $Y$ is a modular pair in $\lambda_{M}$ if and only if $X$ and $Y$ is a modular pair in both $M$ and $M^{*}$.

Proof. It follows from Lemma 2.9 that $X$ and $Y$ is a modular pair in $\lambda_{M}$ if and only if

$$
\begin{aligned}
& r(X)+r^{*}(X)+|X|+r(Y)+r^{*}(Y)+|Y| \\
= & r(X \cup Y)+r^{*}(X \cup Y)+|X \cup Y|+r(X \cap Y)+r^{*}(X \cap Y)+|X \cap Y| .
\end{aligned}
$$

By simplifying, the equation above is equivalent to the following equation,

$$
r(X)+r(Y)+r^{*}(X)+r^{*}(Y)=r(X \cup Y)+r(X \cap Y)+r^{*}(X \cup Y)+r^{*}(X \cap Y) .
$$

Let $X$ and $Y$ be a modular pair in $\lambda_{M}$. Without loss of generality, we assume $X$ and $Y$ is not a modular pair in $M$, that is, $r(X)+r(Y)>r(X \cup Y)+r(X \cap Y)$. Then (3.13) becomes

$$
r^{*}(X)+r^{*}(Y)<r^{*}(X \cup Y)+r^{*}(X \cap Y),
$$


which contradicts the submodularity of rank functions.

If $X$ and $Y$ is a modular pair in both $M$ and $M^{*}$, then it is trivial that $(3.13)$ holds.

The next lemma shows the connectivity function of matroids by deleting and contracting a element from a matroid satisfies property (v) in Lemma 3.7 of being a single-element removal of the connectivity of the given matroid.

Lemma 3.11. Let $M$ be a matroid on $E$ and let $e \in E$. Let $X$ and $Y$ be a modular pair in $\lambda_{M}$. Then the following hold.

(i) If $\lambda_{M \backslash e}(X)=\lambda_{M}(X)-1$ and $\lambda_{M \backslash e}(Y)=\lambda_{M}(Y)-1$, then $\lambda_{M \backslash e}(X \cap Y)=$ $\lambda_{M}(X \cap Y)-1$.

(ii) If $\lambda_{M / e}(X)=\lambda_{M}(X)-1$ and $\lambda_{M / e}(Y)=\lambda_{M}(Y)-1$, then $\lambda_{M / e}(X \cap Y)=$ $\lambda_{M}(X \cap Y)-1$.

Proof. If $\lambda_{M / e}(X)=\lambda_{M}(X)-1, \lambda_{M / e}(Y)=\lambda_{M}(Y)-1$, then by Lemma 3.9. $\lambda_{M / e}(X \cup Y)=\lambda_{M}(X \cup Y)-1$. Since $X$ and $Y$ is a modular pair in $\lambda_{M}$,

$$
\lambda_{M}(X)+\lambda_{M}(Y)=\lambda_{M}(X \cup Y)+\lambda_{M}(X \cap Y) .
$$

Substituting $\lambda_{M / e}(A)=\lambda_{M}(A)-1$ for all $A \in\{X, Y, X \cup Y\}$ into the equation above and simplify, obtain

$$
\lambda_{M / e}(X)+\lambda_{M / e}(Y)=\lambda_{M / e}(X \cup Y)+\lambda_{M}(X \cap Y)-1 .
$$

By the submodularity of $\lambda_{M / e}$ and the equation above,

$$
\lambda_{M / e}(X \cup Y)+\lambda_{M}(X \cap Y)-1 \geq \lambda_{M / e}(X \cup Y)+\lambda_{M / e}(X \cap Y) .
$$

Hence $\lambda_{M}(X \cap Y)-1 \geq \lambda_{M / e}(X \cap Y)$. Equation 3.11 shows $\lambda_{M}(A)-\lambda_{M / e}(A) \leq 1$ for all $A \subseteq E-e$. Therefore (ii) holds. Part (i) holds by the same argument as (ii).

Now we have enough information to show the connectivity function of matroids by deleting and contracting a element from a matroid are both single-element removals of the connectivity of the given matroid.

Lemma 3.12. Let $M$ be a matroid on $E$ and $e \in E$. Then $\lambda_{M \backslash e}$ and $\lambda_{M / e}$ are both single-element removals of e from $\lambda_{M}$.

Proof. It is trivial that both $\lambda_{M \backslash e}$ and $\lambda_{M / e}$ satisfy (i)-(iii) in Lemma 3.7. Lemma 3.9 and Lemma 3.11 show that they satisfy (iv) and (v) in Lemma 3.7 respectively. 


\subsection{The elision operation}

In the last section we have shown that deletion and contraction of an element from a matroid correspond to two single-element removal operations in the connectivity function of the matroid. But an arbitrary unitary connectivity function can not distinguish deletion and contraction, even for a connectivity function of a matroid as $\lambda_{M}=\lambda_{M^{*}}$ and $M / e=\left(M^{*} \backslash e\right)^{*}$ for all $e \in M$, that is deleting $e$ from $M$ gives the same connectivity function as contracting $e$ from $M^{*}$. So it is natural to ask if we can find some operations that always give us a single-element removal from a unitary connectivity function. In this section we find one such operation and it turns out to be important later in this thesis.

Now let us consider Lemma 3.7. Property (iii) shows we have no choice for $\mu^{\prime}(A)$ when $\mu(A) \neq \mu(A \cup e)$. Property (i) shows when $\mu(A)=\mu(A \cup e)$ we have 2 choices for $\mu^{\prime}(A)$, either keep the value or drop the value by one. Therefore we can have many single-element removals from $\mu$ by making different choices for the sets of $A$ such that $\mu(A)=\mu(A \cup e)$, as long as $\mu^{\prime}$ satisfies the remaining properties in Lemma 3.7. But we need these operations to work for all unitary connectivity functions. So we can try the two natural ways which are to keep the value or to drop the value by one for all $A \subseteq E-e$ such that $\mu(A)=\mu(A \cup e)$.

If we drop the value of $\mu(A)$ by one for all $A \subseteq E-e$ such that $\mu(A)=\mu(A \cup e)$, the new function is not always a connectivity function. For example, consider the unitary connectivity function $K_{5,5}$, shown in Table 4.2 , and remove the element $e$ in this way. Then we have a symmetric set function $\mu$ on $\{a, b, c, d\}$ defined as $\mu(a b)=0$ and $\mu(a)=\mu(b)=\mu(c)=\mu(d)=\mu(\{a, c\})=\mu(\{a, d\})=1$. We can see $\mu(a b)+\mu(a c)=1$, but $\mu(a b c)=\mu(a)=\mu(d)+\mu(a)=2$, submodularity does not hold.

Fortunately, the other way of removing the element always gives us a new unitary connectivity function. In this section we study this operation.

Let $\mu$ be a unitary connectivity function on $E$ and let $e \in E$. Define the elision of $e$ from $\mu$, denoted $\mu * e$, by

$$
\mu * e(A)=\left\{\begin{array}{cl}
\min \{\mu(A), \mu(A \cup e)\} & \text { if } \mu(A) \neq \mu(A \cup e), \\
\mu(A) & \text { if } \mu(A)=\mu(A \cup e),
\end{array}\right.
$$

for all $A \subseteq E-e$. It is not hard to see that $\mu * e$ can be defined as the following,

$$
\mu * e(A)=\min \{\mu(A), \mu(A \cup e)\} .
$$

We first show elision from a unitary connectivity always gives a single-element removal.

Lemma 3.13. Let $\mu$ be a unitary connectivity function on $E$ and $e \in E$. Then $\mu * e$ is single-element removal from $\mu$. 
Proof. By Corollary2.4, we have $|\mu(A \cup e)-\mu(A)| \leq 1$. It is trivial that $\mu * e$ satisfies the first three properties of Lemma 3.7. First we show $\mu * e$ satisfies property (iv). Let $A \subseteq E-e$ such that $\mu * e(A)=\mu(A)-1$. By definition of $\mu * e, \mu * e(A)=\min \{\mu(A), \mu(A \cup e)\}$ implies that $\mu(A)>\mu(A \cup e)$. If $B \subseteq E-e$ and $A \subseteq B$, then $\mu(B)>\mu(B \cup e)$ by Lemma 2.5. Hence $\mu * e(B)=\mu(B \cup e)=$ $\mu(B)-1$. Property (iv) holds.

Next we show property (v) holds. Let $\mu(X)+\mu(Y)=\mu(X \cup Y)+\mu(X \cap Y)$. If $\mu * e(X)=\mu(X)-1$ and $\mu * e(Y)=\mu(Y)-1$, by a similar argument as above, we have $\mu(X)>\mu(X \cup e)$ and $\mu(Y)>\mu(Y \cup e)$. Since $X$ and $Y$ is a modular pair in $\mu, \mu(X \cap Y)>\mu((X \cap Y) \cup e)$ by Lemma 2.6. That is $\mu * e(X \cap Y)=$ $\mu((X \cap Y) \cup e)=\mu(X \cap Y)-1$. Property (v) holds.

The next result shows that the order of elision of elements does not matter.

Lemma 3.14. Let $\mu$ be a connectivity function on $E$. Let $e$ and $f$ be two distinct elements of $E$. Then $(\mu * e) * f=(\mu * f) * e$.

Proof. Let $X \subseteq E-\{e, f\}$. By the definition of $*$ operation, $\mu * e(X)=\min \{\mu(X), \mu(X \cup e)\}$ and $\mu * e(X \cup f)=\min \{\mu(X \cup f), \mu(X \cup e \cup f)\}$. So

$$
\begin{aligned}
(\mu * e) * f(X) & =\min \{\mu * e(X), \mu * e(X \cup f)\} \\
& =\min \{\min \{\mu(X), \mu(X \cup e)\}, \min \{\mu(X \cup f), \mu(X \cup e \cup f)\}\} \\
& =\min \{\mu(X), \mu(X \cup e), \mu(X \cup f), \mu(X \cup e \cup f)\}
\end{aligned}
$$

Exchanging $e$ and $f$ and applying the same argument as above, obtain

$$
(\mu * f) * e(X)=\min \{\mu(X), \mu(X \cup f), \mu(X \cup e), \mu(X \cup f \cup e)\}=(\mu * e) * f(X) .
$$

Lemma 3.14 shows that we can remove a set of elements by doing elision without specifying the order in which we apply the operation. Combining with Lemma 3.13 we can see $\mu * X$ is obtained by a sequence of elision of elements in $X$ from a connectivity function $\mu$, where $X \subseteq E$.

\subsection{Elision and extended connectivity functions}

In this section we show that the elision operation and the extended connectivity $\kappa$ coincide.

Let $\mu$ be a unitary connectivity function and let $X$ and $Y$ be disjoint subsets of $E$. Recall from Section 2.3 that the extended connectivity between $X$ and $Y$, denoted $\kappa_{\mu}(X, Y)$, is defined by

$$
\kappa_{\mu}(X, Y)=\min \{\mu(X \cup Z): Z \subseteq E-(X \cup Y)\} .
$$


Let $A \subseteq E$. The function $\kappa_{(\mu, A)}$ on $A$ is defined by

$$
\kappa_{(\mu, A)}(X)=\kappa_{\mu}(X, A-X)
$$

for all $X \subseteq A$. We have shown in Lemma 2.17 that $\kappa_{(\mu, A)}$ is a connectivity function on $A$. We often denote $\kappa_{\mu}(X, Y)$ as $\kappa(X, Y)$ if it is clear from the context which connectivity function we are talking about.

We first show the relation between extended connectivity and the elision operation. Corollary 3.16 follows directly from the following lemma.

Lemma 3.15. Let $\mu$ be a unitary connectivity function on $E$ and let $A \subseteq E$. Let $X \subseteq A$. Then $\kappa(X, A-X)=\mu *(E-A)(X)$.

Proof. This lemma is proved by using induction on $|E-A|$. It is trivial for the case $|E-A|=0$. Assume it holds for $|E-A|=n-1$. Now let $|E-A|=n$ and let $e \in E-A$. We have

$$
\begin{aligned}
& \kappa(X, A-X) \\
= & \min \{\mu(X \cup Z): Z \subseteq E-A\} \\
= & \min \{\mu(X \cup Z), \mu(X \cup Z \cup e): Z \subseteq E-(A \cup e)\} \\
= & \min \{\min \{\mu(X \cup Z): Z \subseteq E-(A \cup e)\}, \min \{\mu(X \cup e \cup Z): Z \subseteq E-(A \cup e)\}\} \\
= & \min \{\kappa(X,(A \cup e)-X), \kappa(X \cup e, A-X)\}
\end{aligned}
$$

By inductive hypothesis, $\kappa(X,(A \cup e)-X)=\mu *(E-(A \cup e))(X)$, and $\kappa(X \cup$ $e, A-X)=\mu *(E-(A \cup e))(X \cup e)$. Substituting these into the equation above, obtain

$$
\begin{aligned}
\kappa(X, A-X) & =\min \{\mu *(E-(A \cup e))(X), \mu *(E-(A \cup e))(X \cup e)\} \\
& =\mu *(E-(A \cup e)) * e(X) \\
& =\mu *(E-A)(X) .
\end{aligned}
$$

The following result follows from Lemma 3.15 directly.

Corollary 3.16. Let $\mu$ be a unitary connectivity function on $E$ and let $A \subseteq E$. Then

$$
\kappa_{(\mu, A)}=\mu *(E-A) .
$$




\section{Chapter 4}

\section{Connectivity Functions on Small Sets}

Let $\mu_{1}$ and $\mu_{2}$ be two connectivity functions on $E_{1}$ and $E_{2}$, respectively. $\mu_{1}$ is isomorphic to $\mu_{2}$ if there is a bijection $\phi$ from $E_{1}$ to $E_{2}$ such that $\mu_{1}(X)=\mu_{2}(\phi(X))$, for all $X \subseteq E_{1}$. A unitary connectivity function is matroidal if it is isomorphic to the connectivity function of a matroid, which was introduced in Section 2.3. It is natural to ask whether every unitary connectivity function is matroidal. In this chapter we will find all the connected unitary connectivity functions on small sets and give some examples of non-matroidal connectivity functions.

In this chapter, it is convenient for us to write the subset $\left\{a_{1}, a_{2}, \ldots, a_{n}\right\}$ as $a_{1} a_{2} \ldots a_{n}$.

\subsection{Connectivity functions on small sets}

In this section, we will find all the connected connectivity functions, up to isomorphism, on sets with size less than 6 , and present the smallest non-matroidal connectivity functions.

For $|E|=1$ the only connected connectivity function is $\mu(\emptyset)=\mu(E)=0$. For $|E|=2,3$, the only connected unitary connectivity function is $\mu(X)=1$ for all $X \subseteq E$ such that $X \notin\{\emptyset, E\}$. So there is only one connected unitary connectivity function on 1-, 2-, or 3-element set. We also know that there are connected matroids on 1-, 2-, and 3-element set. Evidently, every connected unitary connectivity function on 1-, 2-, or 3-element set is matroidal.

We list all the connected unitary connectivity functions on 4-element set in Table 4.1. We will use the following lemma to show that these are all connectivity functions.

Lemma 4.1. Let $\mu$ be a connected symmetric unitary function on a 4-element set $E$. Then $\mu$ is a connectivity function if and only if $\mu(X) \leq|X|$ for all $X \subseteq E$. 
Proof. Suppose $\mu$ is a unitary connectivity function. It follows from the submodularity of $\mu$ that $\mu(X) \leq \sum_{x \in X} \mu(x)=|X|$. Conversely, we show $\mu$ is submodular. It is trivial that if one of $X$ and $Y$ is a subset of another, or $|X \cap Y|=0$, or both $|X|=1$ and $|Y|=1$. So assume neither $X$ nor $Y$ is a subset of another, $|X \cap Y| \neq 0$ and both $|X| \geq 2$ and $|Y| \geq 2$. Let $|X \cap Y|=1$. If $|X \cup Y|=3$, then $1+1=\mu(X \cup Y)+\mu(X \cap Y) \leq \mu(X)+\mu(Y)$. If $X \cup Y=E$, then $1=\mu(X \cap Y)+$ $\mu(X \cup Y) \leq \mu(X)+\mu(Y)$. Submodularity holds for $|X \cap Y|=1$. If $|X \cap Y|=2$ then $|X \cup Y|=4$, hence $2=0+2 \leq \mu(X \cup Y)+\mu(X \cap Y) \leq \mu(X)+\mu(Y)$, submodularity holds in this case. If $|X \cap Y| \geq 3$, then either $X \subseteq Y$ or $Y \subseteq X$, contradicting the assumption. Hence $\mu$ is submodular, which completes the proof.

The following lemma is a direct consequence of Lemma 4.1 .

Lemma 4.2. The connectivity functions in Table 4.1 are all the connected unitary connectivity functions, up to isomorphism, on 4-element set.

\begin{tabular}{|c|c|c|c|c|}
\hline$E=\{a, b, c, d\}$ & $K_{4,1}$ & $K_{4,2}$ & $K_{4,3}$ & $K_{4,4}$ \\
\hline$a b$ & 1 & 1 & 1 & 2 \\
$a c$ & 1 & 1 & 2 & 2 \\
$a d$ & 1 & 2 & 2 & 2 \\
\hline Matroidal & Yes & No & Yes & Yes \\
\hline
\end{tabular}

Table 4.1: Connected connectivity functions on 4-element set

The uniform matroid $U_{r, n}$ is a matroid on a set of $n$ elements with rank function $r$ defined by $r(X)=|X|$ if $|X| \leq r$ and $r(X)=r$ if $|X|>r$, where $r \leq n$.

We give matroids in Figure 4.1 to show $K_{4,1}, K_{4,3}$ and $K_{4,4}$ are all matroidal. In particular, $K_{4,1}$ is the connectivity function of $U_{3,4}$, and $K_{4,4}$ is the connectivity function of $U_{2,4}$.
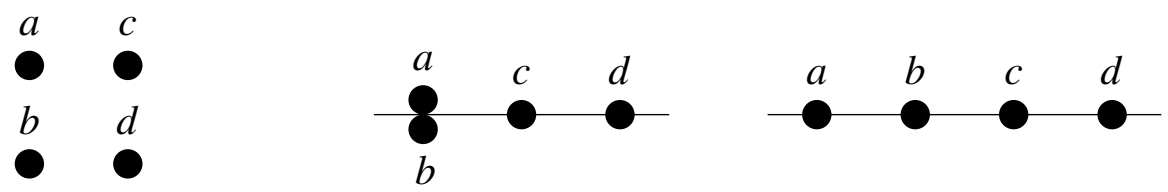

Figure 4.1: The matroids from left to the right have connectivity function $K_{4.1}, K_{4,3}, K_{4,4}$ respectively, where the matroid on the left is $U_{3,4}$, the middle one is the uniform matroid $U_{2,3}$ except that $\{a, b\}$ is a parallel pair instead of a point and the one on the right is $U_{2,4}$.

In the next lemma we show $K_{4,2}$ is a non-matroidal connectivity function. It is the smallest non-matroidal connectivity function.

Lemma 4.3. $K_{4,2}$ is a non-matroidal connectivity function. 
Proof. Suppose there is a matroid $M$ with connectivity function $K_{4,2}$. It is trivial that $r(M) \geq 2$, as $\lambda(a d)=2$. We can see $r(M) \leq 2$, if not, then $r(M)>2$, thus $r^{*}(M)=4-r(M)<2$, contradicition. Therefore we only need to consider the case $r(M)=2$. Since $\lambda(a d)=r(a d)+r(b c)-2=2$, then $r(a d)=r(b c)=2$. Also $\lambda(a b)=r(a b)+r(c d)-2=1$, so $r(a b)=1$ or $r(c d)=1$. Without loss of generality, let $r(a b)=1$. Similarly, $\lambda(a c)=1$ implies $r(a c)=1$ or $r(b d)=1$. Since rank function is submodular and increasing, $r(a b)+r(a c) \geq r(a)+r(a b c) \geq$ $r(a)+r(b c)=3$, then $r(a c)>1$. Similarly $r(a b)+r(b d) \geq r(b)+r(a b d) \geq$ $r(b)+r(b d)=3$, then $r(b d)>1$. Contradicition. Therefore $K_{4,2}$ is not a matroidal connectivity function.

Next we prove a lemma to help us find all the connectivity functions on 5-element set.

Lemma 4.4. Let $E$ be a 5-element set and let $\mu$ be a connected symmetric unitary function on $E$. Then $\mu$ is a connectivity function if and only if the following hold:

(i) $\mu(X) \leq|X|$ for all $X \subseteq E$.

(ii) For any distinct elements $a, b, c, d, e \in E$, if $\mu(\{a, b\})=2$, then at least two of $\mu(\{c, d\}), \mu(\{c, e\}), \mu(\{d, e\})$ are equal to 2 .

Proof. Suppose $\mu$ is a connectivity function. It follows from the submodularity of $\mu$ that (i) holds. Let $X=a b c$ and $Y=a b d$. Then it follows from submodularity of $\mu$ that

$$
\mu(X)+\mu(Y)=\mu(a b c)+\mu(a b d) \geq \mu(a b)+\mu(a b c d)
$$

By symmetry of $\mu$ we have

$$
\mu(E-X)+\mu(E-Y)=\mu(d e)+\mu(c e) \geq \mu(a b)+\mu(e)=3 .
$$

So at least one of $\mu(d e)$ and $\mu(c e)$ equals 2. Let $X=a b c$ and $Y=a b e$. By the same argument, at least one of $\mu(d e)$ and $\mu(c d)$ equals 2 . Similarly $X=a b d$ and $Y=a b e$ implies at least one of $\mu(c e)$ and $\mu(c d)$ equals 2 . As a consequence of the arguments above, at least two of $\mu(c d), \mu(c e)$ and $\mu(d e)$ equal 2.

Conversely, we show that $\mu$ is submodular by checking all the possible cases, that is, for all $X, Y \subseteq E$, the following holds:

$$
\mu(X)+\mu(Y) \geq \mu(X \cup Y)+\mu(X \cap Y) .
$$

It is trivial that 4.1) holds if one of $X$ and $Y$ is a subset of another, or $X$ and $Y$ are disjoint, or either $|X| \leq 1$ or $|Y| \leq 1$. So we assume neither $X$ nor $Y$ is a subset of another, $|X \cap Y| \neq 0$ and both $|X| \geq 2$ and $|Y| \geq 2$. Hence, if $|X|=2$, then $|X \cap Y|=1$, that is, $\mu(X \cap Y)=1$. We can see that 4.1) fails only when $\mu(X)=\mu(Y)=1$ and $\mu(X \cup Y)=2$. As a consequence, $|X \cup Y|=3$ and $|Y|=2$. 
Let $X=c d$ and $Y=d e$. Then $X \cup Y=c d e$. By symmetry of $\mu$, we have $\mu(a b)=2$, but this implies at least two of $\mu(c d), \mu(c e)$ and $\mu(d e)$ equal 2, which means at least one of $\mu(c d)$ and $\mu(d e)$ equals 2 , contradiction. So we assume both $|X| \geq 3$ and $|Y| \geq 3$. If $|X \cap Y|=1$, then $|X \cup Y|=5$. It is trivial that (4.1) holds. So let $|X \cap Y|=2$. For the case $|X|=3$ and $|Y| \geq 4$, we have $|X \cup Y|=5$, that is, $\mu(X \cup Y)=0$, it is easy to see (4.1) holds. So only consider the case $|X|=|Y|=3$, we have $|X \cup Y|=4$, which means $\mu(X \cup Y)=1$. Therefore (4.1) fails only when $\mu(X)=\mu(Y)=1$ and $\mu(X \cap Y)=2$. Let $X=a b c$ and $Y=a b d$. Then $X \cap Y=a b$. We know that $\mu(a b)=2$ implies at least two of $\mu(c d), \mu(c e)$ and $\mu(d e)$ equal 2, which means one of $\mu(d e)$ and $\mu(c e)$ equals 2 . By symmetry of $\mu$, at least one of $\mu(a b c)$ and $a b d$ equals 2 , contradiction. For the case $|X| \geq 4$ and $|Y| \geq 4$, it is trivial that 4.1 holds. Therefore $\mu$ is submodular, which complete the proof.

Now we can find all the connected unitary connectivity functions on 5-element set, which is shown in Table 4.2 .

Lemma 4.5. The connectivity functions in Table 4.2 are all the connected unitary connectivity function, up to isomorphism, on 5-element set.

Proof. For a connected unitary connectivity function with ground set $E$ such that $|E|=5$, the values on singleton are fixed, by symmetry the values on 4-element set are also fixed. By symmetry knowing all the values on 2-element sets will determine the connectivity function $\mu$. By Lemma 4.4 (i), for each 2-element set $A \subseteq E$, either $\mu(A)=1$ or $\mu(A)=2$. So we represent each connectivity function with a graph $G$ such that for all $x, y \in V(G), x$ is adjacent with $y$ if and only if $\mu(\{x, y\})=2$.

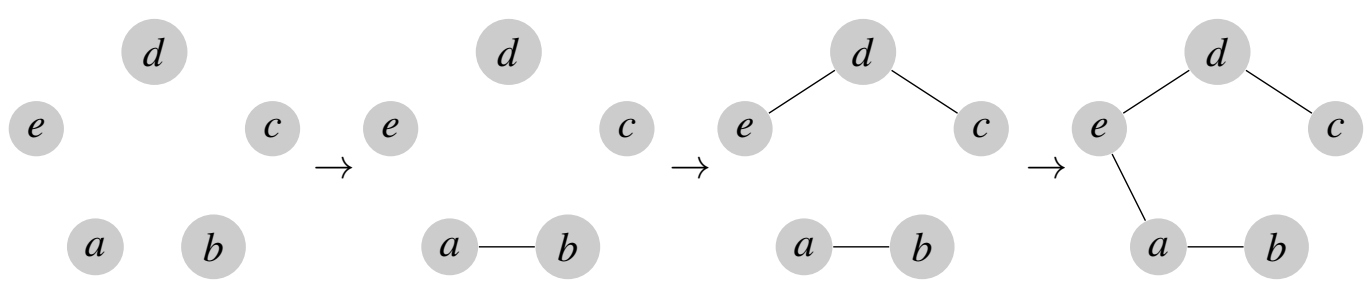

Figure 4.2:

It is clear if $G$ has no edge, then it satisfies Lemma 4.4. So we obtain a connectivity function, call it $K_{5,1}$ as shown in Table 4.2 .

Let $a b$ be an edge of $G$. In order to satisfy Lemma 4.4, we need at least two more edges in the triangle $c d e$, without loss of generality, say $c d$ and $d e$. To satisfy Lemma 4.4, $c d$ is an edge of $G$ implies $a e$ or be must be an edge of $G$, without loss of generality, say $a e$ is an edge of $G$. Similarly, $d e$ is an edge of $G$ implies $a c$ or $b c$ must be an edge of $G$. Hence we have two cases.

Case 1: If $b c$ is an edge of $G$, called it $G_{5}$ as shown in the figure below, then Lemma 4.4 holds. Call the corresponding connectivity function $K_{5,2}$. 


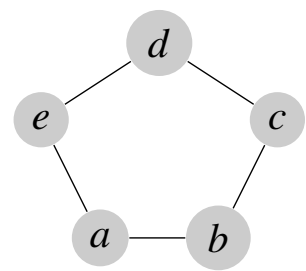

Figure 4.3: $G_{5}$

Case 2: If $a c$ is an edge of $G$, then by Lemma 4.4, either $b d$ or $b e$ is an edge of $G$. If $b d$ is an edge of $G$, called it $G_{6}$ as shown in figure below, then it is easy to see Lemma 4.4 holds. Call the corresponding connectivity function $K_{5,3}$. If $b e$ is an edge of $G$, because $a e$ is an edge, by Lemma 4.4, either $b d$ or $b c$ is an edge of $G$. So $G$ has more than 6 edges, and will be consider later.

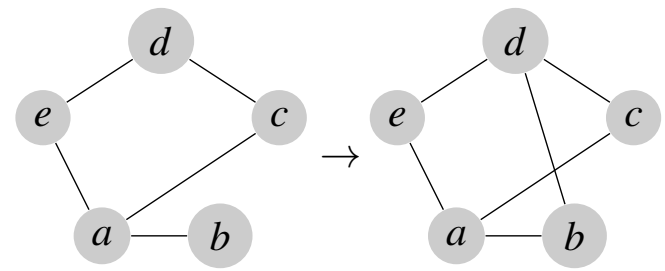

Figure 4.4: $G_{6}$

We have shown that $G_{5}$ is the only graph with 5 edges that satisfies Lemma 4.4 It is not hard to see that adding any other edge in $G_{5}$ will force us to add more edges, otherwise Lemma 4.4 will not hold. Therefore $G_{6}$ is the only graph with 6 edges that satisfies Lemma 4.4 .

Let $G_{7}$ be a graph with 7 edges and satisfies Lemma 4.4. Then the complement $\bar{G}_{7}$ has 3 edges. Because $G_{7}$ has 5 vertices only, two edges in $\bar{G}_{7}$ must incident with a commom vertex. Let $c d$ and $d e$ be these two edges and is shown in the figure below. Then $a b$ must be an edge in $\bar{G}_{7}$. If not, $a b$ will be an edge in $G_{7}$, for Lemma 4.4 to hold at least one of $c d$ and $d e$ is an edge in $G_{7}$, contradicting both $c d$ and $d e$ are edges in $\bar{G}_{7}$. We can check $G_{7}$ satisfies Lemma 4.4 and the argument above shows $G_{7}$ is the only graph with 7 edges that satisfies Lemma 4.4 Call the corresponding connectivity function $K_{5,4}$.

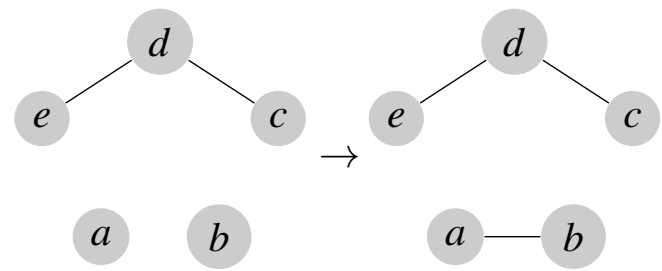

Figure 4.5: $\bar{G}_{7}$ 
Let $G_{8}$ be a graph with 8 edges that satisfies Lemma 4.4 . Then $\bar{G}_{8}$ has 2 edges, the similar argument as above shows that they cannot have a common vertex since there is no third edge to make Lemma 4.4 hold. $G_{8}$ is shown in the figure below and it is not hard to show that it satisfies Lemma 4.4. Call the corresponding connectivity function $K_{5,5}$.

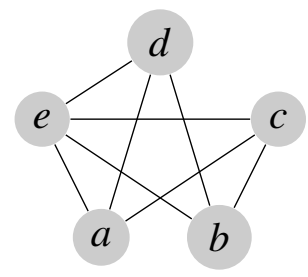

Figure 4.6: $G_{8}$

Let $G_{9}$ be a graph with 9 edges. Then it is isomorphic to $K_{5} \backslash e$ as show below. It is easily to see from the figure below that it satisfies Lemma 4.4. Call the corresponding connectivity function $K_{5,6}$.

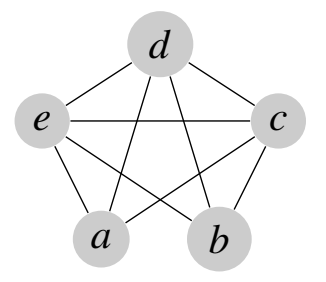

Figure 4.7: $G_{9}$

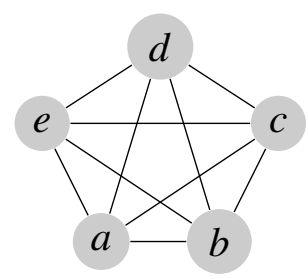

Figure 4.8: $G_{10}$

If $G_{10}$ is a graph with 10 edges, then it is isomorphic to the complete graph $K_{5}$. It satisfies Lemma 4.4. Call the corresponding connectivity function $K_{5,7}$.

In the rest of this section, we generalise the result of Lemma 4.3 to Lemma 4.7 which is a sufficient condition for a connectivity function to be non-matroidal and will be used later in Section 4.2. In particular, Lemma 4.7 immediately implies $K_{5,2}$ and $K_{5,4}$ are non-matroidal connectivity functions.

Let $M$ be a matroid on $E$ and let $X, Y \subseteq E$. The local connectivity between $X$ and $Y$, denoted $\sqcap_{M}(X, Y)$, is defined by $\sqcap_{M}(X, Y)=r(X)+r(Y)-r(X \cup Y)$. We can see $\sqcap_{M}(X, Y) \geq 0$ for all $X, Y \subseteq E$, since the rank function $r$ is submodular. 


\begin{tabular}{|c|c|c|c|c|c|c|c|}
\hline$E=\{a, b, c, d, e\}$ & $K_{5,1}$ & $K_{5,2}$ & $K_{5,3}$ & $K_{5,4}$ & $K_{5,5}$ & $K_{5,6}$ & $K_{5,7}$ \\
\hline$a b$ & 1 & 2 & 2 & 1 & 1 & 1 & 2 \\
$a c$ & 1 & 1 & 2 & 2 & 2 & 2 & 2 \\
$a d$ & 1 & 1 & 1 & 2 & 2 & 2 & 2 \\
$a e$ & 1 & 2 & 2 & 2 & 2 & 2 & 2 \\
$b c$ & 1 & 2 & 1 & 2 & 2 & 2 & 2 \\
$b d$ & 1 & 1 & 2 & 2 & 2 & 2 & 2 \\
$b e$ & 1 & 1 & 1 & 2 & 2 & 2 & 2 \\
$c d$ & 1 & 2 & 2 & 1 & 1 & 2 & 2 \\
$c e$ & 1 & 1 & 1 & 2 & 2 & 2 & 2 \\
$d e$ & 1 & 2 & 2 & 1 & 2 & 2 & 2 \\
\hline Matroidal & Yes & No & Yes & No & Yes & Yes & Yes \\
\hline
\end{tabular}

Table 4.2: Connected connectivity functions on 5-element set

The following lemma (Oxley, Semple, and Whittle [6] ) gives useful link between the local connectivity and the connectivity function of a matroid.

Lemma 4.6. Let $X$ and $Y$ be disjoint subsets of the ground set of a matroid $M$. Then

$$
\sqcap_{M}(X, Y)+\sqcap_{M^{*}}(X, Y)=\lambda(X)+\lambda(Y)-\lambda(X \cup Y) .
$$

Proof. The result follows easily by substitution using the facts that $X$ and $Y$ are disjoint and Lemma 2.9.

Lemma 4.7. Let $\lambda$ be a unitary connectivity function on $E$. If there is a partition $\{A, B, C, D\}$ of $E$ such that $\lambda(A)=\lambda(B)=\lambda(C)=\lambda(D)=\lambda(A \cup B)=\lambda(A \cup C)=$ 1 , and $\lambda(A \cup D)=2$, then $\lambda$ is non-matroidal.

Proof. In this proof, we denote $X \cup Y$ by $X Y$ whenever $X$ and $Y$ are disjoint subsets of $E$. Assume $M$ is a matroid with $r(M)=n$ and connectivity function $\lambda$ which satisfies the properties in the lemma. By Lemma 4.6. $\sqcap_{M}(A, D)+\sqcap_{M^{*}}(A, D)=0$, therefore we have $\sqcap_{M}(A, D)=\sqcap_{M^{*}}(A, D)=0$. By definition of local connectivity

$$
r(A)+r(D)=r(A D) .
$$

The same argument also shows

$$
r(B)+r(C)=r(B C) .
$$

Substituting Equations (4.2) and (4.3) into $\lambda(A D)=r(A D)+r(B C)-r(M)=2$, obtain

$$
r(A)+r(B)+r(C)+r(D)=n+2 .
$$

Since $\lambda(A)=1$,

$$
r(A)+r(B C D)=n+1 .
$$


Combining (4.4), (4.5) and (4.3), obtain

$$
r(B C)+r(D)=r(B C D)+1 .
$$

By Lemma 4.6. $\sqcap_{M}(A, B)+\sqcap_{M^{*}}(A, B)=1$, that is, we have either $\sqcap_{M}(A, B)=0$ or $\sqcap_{M^{*}}(A, B)=0$, without loss of generality, say $\sqcap_{M}(A, B)=0$, hence

$$
r(A)+r(B)=r(A B) .
$$

We show that exactly one of $\sqcap_{M}(A, B)$ and $\sqcap_{M}(C, D)$ is 1 and the other is 0 . Suppose both $\sqcap_{M}(A, B)$ and $\sqcap_{M}(C, D)$ are 0 , then $r(A)+r(B)+r(C)+r(D)=n+1$ as $\lambda(A)=1$, contradicting (4.4). Hence $\sqcap_{M}(C, D)=1$, therefore

$$
r(C)+r(D)=r(C D)+1 .
$$

By the same argument as above, we have either $\sqcap_{M}(A, C)=0$ and $\sqcap_{M}(B, D)=1$, or $\sqcap_{M}(A, C)=1$ and $\sqcap_{M}(B, D)=0$. Without loss of generality, assume the former, then

$$
\begin{gathered}
r(A)+r(C)=r(A C), \\
r(B)+r(D)=r(B D)+1 .
\end{gathered}
$$

By (4.8) and (4.10), we have

$$
r(B)+r(C)+2 r(D)=r(C D)+r(B D)+2 .
$$

Applying (4.3) and (4.6) to the left-hand-side of the equation above and submodularity to the right hand of the equation above, obtain

$$
r(B C D)+r(D) \geq r(B C D)+r(D)+1 .
$$

Contradiction.

The following lemmas are direct results of Lemma 4.7 by finding the right partition of ground set of the connectivity functions.

Corollary 4.8. $K_{5,2}$ is a non-matroidal connectivity function.

Proof. Let $A=\{a\}, B=\{d\}, C=\{c, e\}, D=\{b\}$. Then lemma holds by applying Lemma 4.7 .

Corollary 4.9. $K_{5,4}$ is a non-matroidal connectivity function.

Proof. Let $A=\{a, b\}, B=\{c\}, C=\{e\}, D=\{d\}$. Then lemma holds by applying Lemma 4.7 .

In fact $K_{5,2}$ and $K_{5,4}$ are the only non-matroidal unitary connected connectivity function on 5 -element set. It is easy to see that $K_{5,1}$ is the connectivity function of $U_{1,5}$ and $K_{5,7}$ is the connectivity of $U_{2,5}$. We show $K_{5,3}, K_{5,5}, K_{5,6}$ are matroidal by giving matroids in Figure 4.1 . 

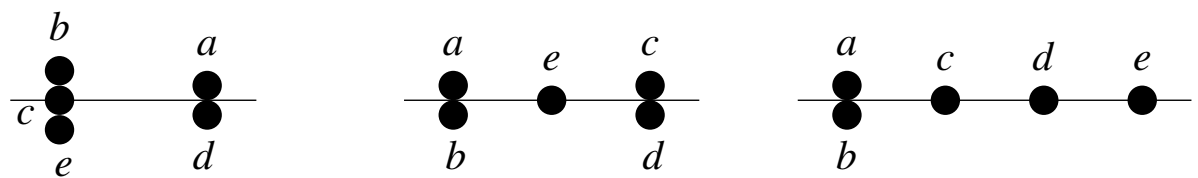

Figure 4.9: The matroids from left to the right have connectivity function $K_{5,3}, K_{5,5}, K_{5,6}$ respectively. The one on the left is the uniform matroid $U_{2,2}$ except that $\{b, c, e\}$ is a parallel class and $\{a, b\}$ is a parallel pair, the middle one is the uniform matroid $U_{2,3}$ except that $\{a, b\}$ is a parallel pair and $\{c, d\}$ is a parallel pair, and the one on the right is the uniform matroid $U_{2,4}$ except that $\{a, b\}$ is a parallel pair.

\subsection{The elision operation and matroidal connectiv- ity functions}

We have shown that $K_{4,2}$ is not matroidal. However the question remains as to whether it can be obtained from a matroid by the elision operation. In this section we show that this cannot happen. We also show that the class of matroidal connectivity functions is not closed under the elision operation by giving a counter example.

Lemma 4.10. Let $\mu$ be a connected unitary connectivity function on $E$. If $\mu * X$ is isomorphic to $K_{4,2}$ for some $X \subseteq E$, then there exist a partition $\{A, B, C, D\}$ of $E$ such that $\mu(A)=\mu(B)=\mu(C)=\mu(D)=\mu(A \cup B)=\mu(A \cup C)=1$ and $\mu(A \cup D)=2$.

Proof. In this proof, we denote $X \cup Y$ by $X Y$ if $X$ and $Y$ are disjoint subsets of $E$. Let $\mu$ be a minimal counter example with respect to the size of the ground set $E$. That is, $\mu * X$ is isomorphic to $K_{4,2}$ for some $X \subseteq E$, but there is no partition of $E$ as described in the lemma. Recall that the order of elision does not matter. Let $e \in X$. By the definition of elision operation, we can easily see that $\mu * e$ is connected and unitary. Then by the minimality of the $\mu$, we know $\mu * e$ is a connectivity function on $E-e$ such that $(\mu * e) *(X-e)$ is isomorphic to $K_{4,2}$, and there exist a partition $\{A, B, C, D\}$ of $E-e$ such that $\mu * e(A)=\mu * e(B)=$ $\mu * e(C)=\mu * e(D)=\mu * e(A B)=\mu * e(A C)=1$ and $\mu * e(A D)=2$.

Suppose $\mu(A)=2$, then $\mu(B C D)=\mu(A \cup e)=1=\mu * e(B C D)$. By the contrapositive of Lemma 3.7 (iv), since $B$ is a subset of $B C D$, we have $\mu(B) \neq \mu * e(B)+1$, that is, $\mu(B)=\mu * e(B)=1$. Similarly, $\mu(C)=\mu(D)=1$. Then we can choose the partition $(A \cup e, B, C, D)$. By applying the contrapositive of Lemma 3.7 (iv), we have $\mu(A B \cup e)=\mu(A C \cup e)=1$ and $\mu(A D \cup e)=2$, so the lemma holds.

Now we assume $\mu(A)=\mu(B)=\mu(C)=\mu(D)=1$. It follows from the submodularity of connectivity functions that $\mu(A D) \leq \mu(A)+\mu(D)=2$ and $\mu(B C) \leq$ $\mu(B)+\mu(C)=2$. If either $\mu(A D)=1$ or $\mu(B C)=1$ then by the definition of $\mu * e$ and the symmetry of $\mu$, we have $\mu * e(A D)=\mu * e(B C)=1$, contradiction. 
So $\mu(A D)=\mu(B C)=2$.

As $\mu * e(A B)=1$, we have $\mu(A B)=1$ or $\mu(C D)=1$. Without loss of generality, let $\mu(A B)=1$. Also $\mu * e(A C)=1$ implies either $\mu(A C)=1$ or $\mu(B D)=1$. If $\mu(A C)=1$, let $A^{\prime}=A, B^{\prime}=B, C^{\prime}=C$ and $D^{\prime}=D \cup e$. Then $\mu\left(A^{\prime}\right)=\mu\left(B^{\prime}\right)=$ $\mu\left(C^{\prime}\right)=\mu\left(D^{\prime}\right)=\mu\left(A^{\prime} B^{\prime}\right)=\mu\left(A^{\prime} C^{\prime}\right)=1$ and $\mu\left(A^{\prime} D^{\prime}\right)=2$. Contradicting $\mu$ is a counter example. So let $\mu(B D)=1$. We have a partition $\left\{A^{\prime}, B^{\prime}, C^{\prime}, D^{\prime}\right\}$ of $E$ where $A^{\prime}=A, B^{\prime}=B, C^{\prime}=C \cup e$ and $D^{\prime}=D$, such that $\mu\left(A^{\prime}\right)=\mu\left(B^{\prime}\right)=\mu\left(C^{\prime}\right)=$ $\mu\left(D^{\prime}\right)=\mu\left(A^{\prime} B^{\prime}\right)=\mu\left(A^{\prime} C^{\prime}\right)=1$ and $\mu\left(A^{\prime} D^{\prime}\right)=2$. Contradicting $\mu$ is a counter example. Therefore the counter example does not exist.

Combining Lemma 4.10 and Lemma 4.7 we obtain the following lemma directly.

Lemma 4.11. Let $\mu$ be a unitary connectivity function on $E$. If $\mu * X$ is isomorphic to $K_{4,2}$ for some $X \subset E$, then $\mu$ is non-matroidal.

The following lemma shows that the class of matroidal connnectivity functions is not closed under the elision operation.

Lemma 4.12. There exist a matroid $M$ on $E$ such that $\lambda_{M} * e$ is not a matroidal connectivity function, where $e \in E$.

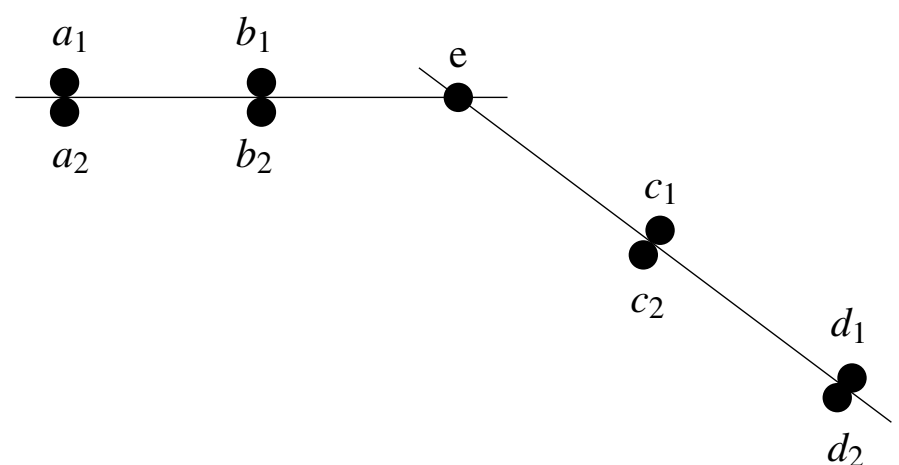

Figure 4.10: A matroid such that the elision of the element $e$ from its connectivity function is non-matroidal.

Proof. Consider the matroid $M$ in Figure 4.2, we show that $\lambda_{M} * e$ is non-matroidal. For convenience, in this proof we will use $\mu$ to denote $\lambda * e$, use $x y z$ to denote the set $\{x, y, z\}$. For $x \in\{a, b, c, d\}$, we use $x$ to denote any one of $x_{1}$ and $x_{2}$, for instance, $r(a)=1$ means for all $a \in\left\{a_{1}, a_{2}\right\}, r(a)=1$, and $\lambda(a b)=2$ means for all $a \in\left\{a_{1}, a_{2}\right\}$ and for all $b \in\left\{b_{1}, b_{2}\right\}, \lambda(a b)=2$, etc.

It is not hard to see that the connectivity function $\mu$ is

$$
\mu(X)= \begin{cases}1 & \text { if } X \text { or } E^{\prime}-X \text { is in } E_{1} \cup A \cup\left\{a_{1} a_{2} b_{1} b_{2}\right\}, \\ 2 & \text { if } X \text { or } E^{\prime}-X \text { is in } E_{2} \cup B \cup\left\{a_{1} a_{2} c_{1} c_{2}, a_{1} a_{2} d_{1} d_{2}\right\}-A, \\ 3 & \text { else, }\end{cases}
$$

where $E^{\prime}=E-e$,

$E_{n}=\{X:|X|=n\}$ for $n=\{1,2\}$, 
$A=\left\{a_{1} a_{2}, b_{1} b_{2}, c_{1} c_{2}, d_{1} d_{2}\right\}$,

$B=\left\{x \cup y: x \in E_{1}, y \in A,|x \cup y|=3\right\}$.

Suppose $\mu$ is a connectivity function of a matroid. Since $|E-e|=8$ and the maximum connectivity value of a subset of $E-e$ is 3 , up to duality we only need to check the cases $r(M)=3$ and $r(M)=4$.

Suppose $r(M)=3$. Since $\mu(a b)=\mu(c d)=2, r\left(a_{1} a_{2} b_{1} b_{2}\right) \geq 2$ and $r\left(c_{1} c_{2} d_{1} d_{2}\right) \geq$ 2 . Hence we can see $\mu\left(a_{1} a_{2} b_{1} b_{2}\right)=r\left(a_{1} a_{2} b_{1} b_{2}\right)+r\left(c_{1} c_{2} d_{1} d_{2}\right)-3=1$ implies that

$$
r\left(a_{1} a_{2} b_{1} b_{2}\right)=r\left(c_{1} c_{2} d_{1} d_{2}\right)=2 .
$$

As rank function is increasing it follows from (4.14) that $r\left(a_{1} a_{2} b_{1}\right) \leq 2$ and since $\mu\left(a_{1} a_{2} b_{1}\right)=2$, we have $r\left(a_{1} a_{2} b_{1}\right)=2$ and $r\left(b_{2} c_{1} c_{2} d_{1} d_{2}\right)=3$ which implies that $r\left(b_{1} b_{2} c_{1} c_{2} d_{1} d_{2}\right)=3$. Therefore $\mu\left(a_{1} a_{2}\right)=1$ implies $r\left(a_{1} a_{2}\right)=1$. Similarly, we have $r\left(b_{1} b_{2}\right)=r\left(c_{1} c_{2}\right)=r\left(d_{1} d_{2}\right)=1$.

As rank function is submodular, we have $r\left(a_{1} a_{2} c_{1} c_{2}\right) \leq 2$ and $r\left(b_{1} b_{2} d_{1} d_{2}\right) \leq 2$. Hence $\mu\left(a_{1} a_{2} c_{1} c_{2}\right) \leq 2+2-3=1 \neq 2$, contradiction.

Suppose $r(M)=4$. For any $X \subseteq E$ such that $|X|=3$ and $\mu(X)=3$, we have $r(X)=3$. So $\mu(a b c)=3$ implies

$$
r(a b c)=3
$$

As a consequence of 4.15) $r(a b)=2$. Hence we have $r\left(a_{1} a_{2} b_{1} b_{2}\right) \geq 2$. Similarly, $\mu($ acd $)=3$ implies $r\left(c_{1} c_{2} d_{1} d_{2}\right) \geq 2$. As $\mu\left(a_{1} a_{2} b_{1} b_{2}\right)=1$, either one of $\mu\left(a_{1} a_{2} b_{1} b_{2}\right)$ and $\mu\left(c_{1} c_{2} d_{1} d_{2}\right)$ has value 2 and the other has value 3 . Without loss of generality say

$$
r\left(a_{1} a_{2} b_{1} b_{2}\right)=2 \quad \text { and } \quad r\left(c_{1} c_{2} d_{1} d_{2}\right)=3 .
$$

By the properties of rank function and the equations above we have

$$
2 \leq r\left(a_{1} a_{2} b_{1} b_{2} c_{1}\right) \leq 3 \quad \text { and } \quad 2 \leq r\left(c_{2} d_{1} d_{2}\right) \leq 3
$$

It follows from $\mu\left(c_{2} d_{1} d_{2}\right)=2$ that $r\left(a_{1} a_{2} b_{1} b_{2} c_{1}\right)+r\left(c_{2} d_{1} d_{2}\right)=6$. Combining this with 4.17), we obtain $r\left(c_{2} d_{1} d_{2}\right)=r\left(a_{1} a_{2} b_{1} b_{2} c_{1}\right)=3$. As a consequence, $r\left(d_{1} d_{2}\right)=2$. Therefore we have

$$
r\left(a_{1} a_{2} b_{1} b_{2} c_{1} c_{2}\right)=\mu\left(d_{1} d_{2}\right)+r(M)-r\left(d_{1} d_{2}\right)=1+4-2=3 .
$$

Since $\mu\left(d_{1}\right)=\mu\left(d_{2}\right)=1$, it is not hard to see

$$
r\left(a_{1} a_{2} b_{1} b_{2} c_{1} c_{2} d\right)=4 .
$$

By submodularity of $r$ and Equations 4.15, 4.19) and 4.18), we have $r\left(a_{1} b_{1} c_{1} d_{1}\right) \geq r\left(a_{1} b_{1} c_{1}\right)+r\left(a_{1} a_{2} b_{1} b_{2} c_{1} c_{2} d_{1}\right)-r\left(a_{1} a_{2} b_{1} b_{2} c_{1} c_{2}\right)=3+4-3=4$. 
$r\left(a_{2} b_{2} c_{2} d_{2}\right) \geq r\left(a_{2} b_{2} c_{2}\right)+r\left(a_{1} a_{2} b_{1} b_{2} c_{1} c_{2} d_{2}\right)-r\left(a_{1} a_{2} b_{1} b_{2} c_{1} c_{2}\right)=3+4-3=4$.

But, $\mu\left(a_{1} b_{1} c_{1} d_{1}\right)=3$ which means $r\left(a_{1} b_{1} c_{1} d_{1}\right)+r\left(a_{2} b_{2} c_{2} d_{2}\right)=7<8$, a contradiction. 


\section{Chapter 5}

\section{Connectivity Functions and Polymatroids}

So far we have defined polymatroids to be integer-valued, but in this chapter it is convenient to extend the definition. A fractional polymatroid $P$ on $E$ is an ordered pair $(E, r)$, where $E$ is a finite set and $r: 2^{E} \rightarrow \mathbb{Q}^{+} \cup\{0\}$ is a set function that is normalised increasing and submodular. The fractional polymatroid on $E$ is halfintegral if $r(A) \in\left\{\frac{n}{2}: n \in \mathbb{Z}^{+} \cup\{0\}\right\}$ for all $A \subseteq E$. From now on we will refer to a polymatroid that takes only integer values as an integer polymatroid. Recall that a $k$-polymatroid $P=(E, r)$ is an integer polymatroid such that $r(e) \leq k$ for all $e \in E$. A $k$-polymatroid is strict if $r(e)=k$ for all $e \in E$.

Let $P$ be a fractional polymatroid on $E$. We define the connectivity function of $P$, denoted $\lambda_{P}$, by

$$
\lambda_{P}(X)=r(X)+r(E-X)-r(E)
$$

for all $X \subseteq E$. Strictly speaking, $\lambda_{P}$ may not be a connectivity function as we have insisted that connectivity functions are integer-valued. However, it may be the case that $\lambda_{P}$ is integer-valued (and hence a connectivity function) even when $P$ is not integer-valued. We give an example below.

Example 5.1. Let $G$ be a graph as shown in Figure 5.1. Then $\lambda_{G}(a)=\lambda_{G}(b)=$ $\lambda_{G}(c)=\lambda_{G}(d)=\lambda_{G}(a b)=\lambda_{G}(a c)=2$ and $\lambda_{G}(a d)=4$. It is easy to see that $\mu$ defined by $\mu(X)=\frac{1}{2} \lambda_{G}(X)$ for all $X \subseteq E$ is also a connectivity function on $E$. More specifically, $\mu=K_{4,2}$, where $K_{4,2}$ is the non-matroidal unitary connectivity as shown in Table 4.1

Let $P$ be a fractional polymatroid on $\{a, b, c, d\}$ with $r$ defined as

$$
r(X)= \begin{cases}1 & \text { if } X \in\{\{a\},\{b\},\{c\},\{d\}\}, \\ \frac{3}{2} & \text { if } X \in\{\{a, b\},\{b, d\},\{c, d\},\{a, c\}\} \\ 2 & \text { else. }\end{cases}
$$

Then the connectivity function of $P$ is $\lambda_{P}(a)=\lambda_{P}(b)=\lambda_{P}(c)=\lambda_{P}(d)=\lambda_{P}(a b)=$ $\lambda_{P}(a c)=1$ and $\lambda_{P}(a d)=2$. That is $\lambda_{P}=K_{4,2}$. Hence $\lambda_{P}$ is integer-valued. 


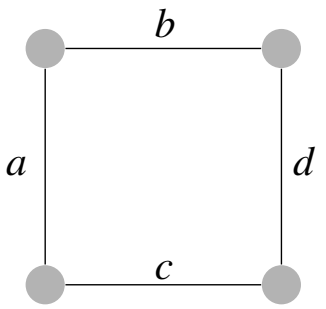

Figure 5.1:

We have shown in Section 4.1 that not every unitary connectivity function is the connectivity function of a matroid. It is interesting to ask if there are natural strucures that give us all connectivity functions. In this chapter we answer this question in the affirmative by proving that every connectivity function is the connectivity function of a half-integral polymatroid. In particular, we prove

Theorem 5.2. Let $\mu$ be a connectivity function on $E$. Then there is a half-integral polymatroid $P$ on $E$ such that $\mu=\lambda_{P}$.

We also give related results giving more specific details about the structure of the polymatroids. To do this we will need to derive certain types of polymatroids.

\subsection{Proof of the main theorem}

Let $P$ be an integer polymatroid on $E$ and let $e \in E$. We say $e$ is compact if $r_{P}(e)=\lambda_{P}(e)$, and the integer polymatroid $P$ is compact if every element of $P$ is compact.

In this section we first show that for any integer polymatroid $P$ there is a compact integer polymatroid $P^{\prime}$ such that $\lambda_{P}=\lambda_{P^{\prime}}$. And then we prove the main result.

Let $\mu$ be a connectivity function on $E$ and let $X \subseteq E$. We define

$$
\|X\|_{\mu}=\sum_{x \in X} \mu(x)
$$

We write $\|X\|_{\mu}$ as $\|X\|$ if $\mu$ is clear from the context.

The following lemma shows how to construct a compact polymatroid from an integer polymatroid without changing the connectivity function.

Lemma 5.3. Let $P=(E, r)$ be an integer polymatroid, and $e \in E$ is not compact. Define $r^{\prime}$ on $E$ by

$$
r^{\prime}(X)= \begin{cases}r(X) & \text { if } e \notin X \\ r(X)+\lambda_{P}(e)-r(e) & \text { if } e \in X\end{cases}
$$

for all $X \subseteq E$. Then the following hold. 
(i) $P^{\prime}=\left(E, r^{\prime}\right)$ is an integer polymatroid;

(ii) $\lambda_{P^{\prime}}=\lambda_{P}$;

(iii) e is compact in $P^{\prime}$.

Proof. (i) It is trivial $r^{\prime}$ is integer-valued. Let $X, Y, Z \subseteq E$ such that $X \subseteq Y$. It is trivial that $r^{\prime}(\emptyset)=r(\emptyset)=0$. We show $r^{\prime}$ is increasing. If $e \notin Y$, then $r^{\prime}(X)=$ $r(X) \leq r(Y)=r^{\prime}(Y)$. If $e \in X$, then $r^{\prime}(X)=r(X)+\lambda(e)-r(e) \leq r(Y)+\lambda(e)-$ $r(e)=r^{\prime}(Y)$. If $e \in Y$ but $e \notin X$, assume $r^{\prime}(X)>r^{\prime}(Y)$ then $r(X)>r(Y)+\lambda(e)-$ $r(e)=r(Y)+r(e)+r(E-e)-r(E)-r(e)$. This implies $r(X)+r(E)>r(Y)+$ $r(E-e)$. By submodularity of $r$, we have $r(X)+r(E)>r(Y-e)+r(E)$, thus $r(X)>r(Y-e)$ contradiction. Next we show $r^{\prime}$ is submodular. Observe that if $e$ is in both $X$ and $Z$ then $e$ is in both $X \cup Z$ and $X \cap Z$. If $e$ is in exactly one of $X$ and $Z$ then $e$ is in exactly one of $X \cup Z$ and $X \cap Z$. If $e$ is not in both $X$ and $Z$ then $e$ is not in both $X \cup Z$ and $X \cap Z$. Let $k=|X \cup e-X|+|Z \cup e-Z|$. Then

$$
\begin{aligned}
r^{\prime}(X)+r^{\prime}(Z) & =r(X)+r(Z)+k\left(\lambda_{r}(e)-r(e)\right) \\
& \geq r(X \cup Z)+r(X \cap Z)+k\left(\lambda_{r}(e)-r(e)\right) \\
& =r^{\prime}(X \cup Z)+r^{\prime}(X \cap Z) .
\end{aligned}
$$

(ii) We know $e$ is in exactly one of $X$ and $E-X$ for any $X \subseteq E$. Thus $r^{\prime}(X)+$ $r^{\prime}(E-X)=r(X)+r(E-X)+\lambda_{P}(e)-r(e)$, by the definition of $r^{\prime}$. Therefore

$$
\begin{aligned}
\lambda_{P^{\prime}}(X) & =r^{\prime}(X)+r^{\prime}(E-X)-r^{\prime}(E) \\
& =r(X)+r(E-X)+\lambda_{P}(e)-r(e)-\left(r(E)+\lambda_{P}(e)-r(e)\right) \\
& =r(X)+r(E-X)-r(E) \\
& =\lambda_{P}(X)
\end{aligned}
$$

(iii) By the definition of $r^{\prime}$ and (ii) above we have

$$
r^{\prime}(e)=r(e)+\lambda_{P}(e)-r(e)=\lambda_{P}(e)=\lambda_{P^{\prime}}(e) .
$$

The following corollary is a direct consequence of Lemma 5.3 .

Corollary 5.4. For any integer polymatroid $P$, there is a compact integer polymatroid $P^{\prime}$ such that $\lambda_{P^{\prime}}=\lambda_{P}$.

Now we start to prove our main theorem. The following lemma is elementary.

Lemma 5.5. Let $\mu$ be a connectivity function on $E$. Then the following hold.

(i) $\mu(X) \leq\|X\|$ for all $X \subseteq E$. 
(ii) $\mu(X)-\mu(Y) \leq\|Y-X\|$ for all $X \subseteq Y \subseteq E$.

Proof. Part (i) follows from applying the submodularity of $\mu$ inductively. It is trivial that (ii) holds if $\mu(X) \leq \mu(Y)$, so we assume $\mu(X)>\mu(Y)$. By Lemma 2.2. we have $\mu(X)-\mu(Y) \leq \mu(Y-X)$. Then part (ii) follows from part (i).

Lemma 5.6. Let $\mu$ be a connectivity function on $E$. Define a set function $t$ on $E$ by $t(X)=\mu(X)+\|X\|$ for all $X \subseteq E$. Then $P=(E, t)$ is a compact integer polymatroid. Moreover $\lambda_{P}(X)=2 \mu(X)$ for all $X \subseteq E$.

Proof. It is trivial that $t$ is normalised. Let $X, Y, Z \subseteq E$ such that $X \subseteq Y$. First we show $t$ is increasing. Suppose $t(X)>t(Y)$, that is $\mu(X)+\|X\|>\mu(Y)+\|Y\|$. Then $\mu(X)-\mu(Y)>\|Y\|-\|X\|=\|Y-X\|$, contradicting Lemma 5.5 (ii). We next show $t$ is submodular. As $\mu$ is submodular, then

$$
\begin{aligned}
t(X)+t(Z) & =\mu(X)+\|X\|+\mu(Z)+\|Z\| \\
& \geq \mu(X \cup Z)+\mu(X \cap Z)+\|X \cup Z\|+\|X \cap Z\| \\
& =t(X \cup Z)+t(X \cap Z) .
\end{aligned}
$$

Since $\mu(X)$ and $\|X\|$ are both integers, $t(X)$ is also an integer, for all $X \subseteq E$. Hence $P$ is an integer polymatroid. It follows from the definition of $\lambda_{P}$ that

$$
\begin{aligned}
\lambda_{P}(X) & =t(X)+t(E-X)-t(E) \\
& =\mu(X)+\|X\|+\mu(E-X)+\|E-X\|-\mu(E)-\|E\| \\
& =\mu(X)+\mu(E-X) \\
& =2 \mu(X) .
\end{aligned}
$$

In particular, $\lambda_{P}(e)=2 \mu(e)=\mu(e)+\|e\|=t(e)$ for all $e \in E$. So $P$ is compact.

Lemma 5.7. Let $P$ be an integer polymatroid on $E$ with rank function $r$. Let $r^{\prime}$ be a set function on $E$ defined by $r^{\prime}(A)=\frac{1}{2} r(A)$ for all $A \subseteq E$. Then $P^{\prime}=\left(E, r^{\prime}\right)$ is a half-integral polymatroid.

Proof. It is obvious that $r^{\prime}(\emptyset)=0$. Let $X, Y, Z \subseteq E$ such that $X \subseteq Y$. We show $r^{\prime}$ is increasing. As $r$ is increasing, so $r^{\prime}(X)=\frac{1}{2} r(X) \leq \frac{1}{2} r(Y)=r^{\prime}(Y)$. Next we show $r^{\prime}$ is submodular. As $r$ is submodular, so

$r^{\prime}(X)+r^{\prime}(Z)=\frac{1}{2} r(X)+\frac{1}{2} r(Z) \geq \frac{1}{2}(r(X \cup Z)+r(X \cap Z))=r^{\prime}(X \cup Z)+r^{\prime}(X \cap Z)$.

Since $P$ is an integer polymatroid, $r(A)$ is an integer for all $A \subseteq E$. So $r^{\prime}(A)=$ $\frac{1}{2} r(A) \in\left\{\frac{n}{2}: n \in \mathbb{Q}^{+} \cup\{0\}\right\}$ for all $A \subseteq E$. Hence $P^{\prime}$ is a half-integral polymatroid.

Combining Lemma 5.6 and Lemma 5.7 we have the following corollary, which implies Theorem 5.2 . 
Corollary 5.8. Let $\mu$ be a connectivity function on $E$. Define a set function $r$ by $r(X)=\frac{1}{2}(\mu(X)+\|X\|)$ for all $X \subseteq E$. Then $P^{\prime}=(E, r)$ is a half-integral polymatroid such that $\lambda_{P}=\mu$.

\subsection{Connectivity polymatroids}

In this section we restrict our attention to unitary connectivity functions. We study the 2-polymatroids constructed from unitary connectivity functions as shown in Lemma 5.6, and give some interesting results about the structure of these 2polymatroids. We start with defining this class of polymatroids and then give a characterization of these polymatroids.

Let $\mu$ be a unitary connectivity function on $E$. Then the 2-polymatroid, denoted $P(\mu)$, with rank function $r_{P(\mu)}$ defined by $r_{P(\mu)}(X)=\|X\|_{\mu}+\mu(X)$ for all $X \subseteq E$ is called a connectivity polymatroid generated by $\mu$.

Let $P=(E, r)$ be a $k$-polymatroid. The dual of $P$, denoted by $P^{*}$, is a polymatroid with rank function $r^{*}$ defined as

$$
r_{P}^{*}(X)=k|X|+r_{P}(E-X)-r_{P}(E)
$$

for all $X \subseteq E$. Whittle showed in [9] that this is the only dual operation in $k$ polymatroids that interchanges deletion and contraction, and $\left(r_{P}^{*}\right)^{*}=r_{P}$.

Theorem 5.9. Let $P$ be a strict 2-polymatroid. Then $P$ is a connectivity polymatroid if and only if $P^{*}=P$.

Proof. $P$ is a strict 2-polymatroid, by the definition of $r_{P}^{*}$, we have

$$
r_{P}^{*}(X)=2|X|+r_{P}(E-X)-r_{P}(E) .
$$

If $P$ is a connectivity polymatroid generated by a connected unitary connectivity function $\mu$, then for any $X \subseteq E$, we have

$$
r_{P}(X)=\|X\|_{\mu}+\mu(X)=|X|+\mu(X) .
$$

Applying (5.3) to (5.2) and simplify, obtain

$$
r_{P}^{*}(X)=2|X|+\mu(E-X)+|E-X|-\mu(E)-|E|=|X|+\mu(X)=r_{P}(X) .
$$

Therefore $P^{*}=P$.

Conversely, $r_{P}^{*}(X)=r_{P}(X)$ for all $X \subseteq E$. Rearranging (5.2) we have

$$
r_{P}(E-X)-r_{P}(E)=r_{P}^{*}(X)-2|X|
$$


Hence we have

$$
\begin{aligned}
\lambda_{P}(X) & =r_{P}(X)+r_{P}(E-X)-r_{P}(E) \\
& =r_{P}(X)+r_{P}^{*}(X)-2|X| \\
& =2\left(r_{P}(X)-|X|\right) .
\end{aligned}
$$

Therefore $\lambda_{P}$ takes even values. Obviously, the set function $\mu$ defined by

$$
\mu(X)=\frac{1}{2} \lambda_{P}(X)=r_{P}(X)-|X|
$$

is a unitary connectivity function. Rearranging the equation above, obtain

$$
r_{P}(X)=|X|+\mu(X)=\|X\|_{\mu}+\mu(X) .
$$

Therefore $P$ is a connectivity polymatroid generated by $\mu$.

In Section 3.5 we defined the elision operation in unitary connectivity functions and showed that the class of unitary connectivity functions is closed under elision. Now we define the elision operation for polymatroids and then prove a very interesting result which shows the elision operation in unitary connectivity functions and the elision in connectivity polymatroids coincide. This result immediately implies the class of connectivity functions is closed under elision.

Let $P$ be a 2-polymatroid on $E$, and $l \in E$. The elision of $l$ from $P$, denoted $P * l$, is defined by

$$
r_{P * l}(X)=\left\{\begin{array}{cl}
r_{P}(X)-1 & \text { if } r_{P}(X)=r_{P}(X \cup l) \\
r_{P}(X) & \text { if } r_{P}(X)<r_{P}(X \cup l),
\end{array}\right.
$$

for all $X \subseteq E$. Intuitively, this operation is achieved by adding a point freely on the line $l$ and then contracting the added point (this operation is also called principal truncation, see for example in [5, Section 7.3]).

Theorem 5.10. Let $P(\mu)$ be a connectivity polymatroid on $E$ generated by a unitary connectivity function $\mu$ and let $l \in E$. Then

$$
P(\mu) * l=P(\mu * l) .
$$

Proof. By definition of $\mu * l$, for all $X \subseteq E-l$

$$
\mu * l(X)= \begin{cases}\mu(X) & \text { if } \mu(X) \leq \mu(X \cup l), \\ \mu(X)-1 & \text { if } \mu(X)>\mu(X \cup l) .\end{cases}
$$

Then by the definition of connectivity polymatroids, $r_{P(\mu * l)}(X)=\mu * l(X)+|X|$, we have

$$
r_{P(\mu * l)}(X)=\mu * l(X)+|X|= \begin{cases}\mu(X)+|X| & \text { if } \mu(X) \leq \mu(X \cup l), \\ \mu(X)+|X|-1 & \text { if } \mu(X)>\mu(X \cup l) .\end{cases}
$$


Since $r_{P(\mu)}(X)=\mu(X)+|X|$, the equation above becomes

$$
r_{P(\mu * l)}(X)= \begin{cases}r_{P(\mu)}(X) & \text { if } \mu(X) \leq \mu(X \cup l), \\ r_{P(\mu)}(X)-1 & \text { if } \mu(X)>\mu(X \cup l)\end{cases}
$$

Notice that $\mu(X) \leq \mu(X \cup l)$ if and only if $\mu(X)<\mu(X \cup l)+1$ if and only if $\mu(X)+|X|<\mu(X \cup l)+|X \cup l|$, that is by definition, $r_{P(\mu)}(X)<r_{P(\mu)}(X \cup l)$. Similarly $\mu(X)>\mu(X \cup l)$ if and only if $r_{P(\mu)}(X)=r_{P(\mu)}(X \cup l)$. Hence

$$
r_{P(\mu * l)}(X)= \begin{cases}r_{P(\mu)}(X) & \text { if } r_{P(\mu)}(X)<r_{P(\mu)}(X \cup l), \\ r_{P(\mu)}(X)-1 & \text { if } r_{P(\mu)}(X)=r_{P(\mu)}(X \cup l) .\end{cases}
$$

We know from the definition that

$$
r_{P(\mu) * l}(X)= \begin{cases}r_{P(\mu)}(X) & \text { if } r_{P(\mu)}(X)<r_{P(\mu)}(X \cup l), \\ r_{P(\mu)}(X)-1 & \text { if } r_{P(\mu)}(X)=r_{P(\mu)}(X \cup l) .\end{cases}
$$

Therefore $r_{P(\mu * l)}(X)=r_{P(\mu) * l}(X)$ for all $X \subseteq E-l$.

The following corollary is a direct consequence of Theorem 5.10 .

Corollary 5.11. Let $P$ be a connectivity polymatroid on $E$ and $l \in E$. Then $P * l$ is a connectivity polymatroid.

Finally, let us restrict our attention to matroidal connectivity functions. Notice that if $\mu$ is a connected unitary connectivity function on $E$, then $r_{P(\mu)}(X)=|X|+\mu(X)$ for all $X \subseteq E$.

The following result reduces the interesting question of determining whether a unitary connectivity function is matroidal to a decomposition problem in a 2polymatroid.

Theorem 5.12. Let $\mu$ be a connected unitary connectivity function on $E$ and let $P(\mu)$ be the connectivity polymatroid generated by $\mu$. Then $\mu$ is a matroidal connectivity function if and only if there is a connected matroid $M$ on $E$ such that

$$
r_{P(\mu)}(X)=r_{M}(X)+r_{M^{*}}(X)
$$

for all $X \subseteq E$.

Proof. Suppose $\mu$ is matroidal. Then by definition $\mu=\lambda_{M}$ for some matroid $M$, that is, $r_{P(\mu)}(X)=|| X||+\mu(X)=|X|+\lambda_{M}(X)$ for all $X \subseteq E$. We also know that $\lambda_{M}(X)=r_{M}(X)+r_{M^{*}}(X)-|X|$, so $r_{P(\mu)}(X)=r_{M}(X)+r_{M^{*}}(X)$. The converse holds by reversing the argument above. 


\section{Bibliography}

[1] Clark, B., Whittle, G. Tangles, trees and flowers. J. Combin. Theory Ser. B 103, (2013) 385-407.

[2] Geelen, J., Gerard, B., Whittle, G. Tangles, tree-decompositions and grids in matroids. J. Combin. Theory Ser. B 99, (2009) 657-667.

[3] Lemos, M. Matroids having the same connectivity functions. Discrete Math 131, (1994) 153-161.

[4] Lemos, M. On the connectivity function of a binary matroid. J. Combin. Theory Ser. B 86, (2002) 114-132.

[5] Oxley, J. Matroid Theory. 2nd Edition, Oxford Graduate Texts in Mathematics. Oxford University Press, Oxford, 2011.

[6] Oxley, J., Semple, C., and Whittle, G. The structure of 3-separations of 3connected matroids. J. Combin. Theory Ser. B 92, (2004) 257-293.

[7] Oxley, J., Whittle, G. A characterization of Tutte invariants of 2-polymatroids. J. Combin. Theory Ser. B 59, (1993) 210-244.

[8] Seymour, P.D . On the connectivity function of a matroid. J. Combin. Ser. B 45, (1988) 25-30.

[9] Whittle, G. Duality in Polymatroids and Set Functions. Combinatorics, Probability and Computing 1, (1992) 275-280. 